L. Paris

Nagoya Math. J.

Vol. 131 (1993), 39-65

\title{
THE DELIGNE COMPLEX OF A REAL ARRANGEMENT OF HYPERPLANES
}

\author{
LUIS PARIS
}

\section{Introduction}

Let $V$ be a real vector space. An arrangement of hyperplanes in $V$ is a finite family $\mathscr{A}$ of hyperplanes of $V$ through the origin. We say that $\mathscr{A}$ is essential if $\cap_{H \in \mathscr{A}} H=\{0\}$.

Let $V_{\mathbf{C}}=\mathbf{C} \otimes V$ be the complexification of $V$. Every element $z$ of $V_{\mathbf{C}}$ can be written in a unique way $z=x+i y$, where $x, y \in 1 \otimes V=V$. We say that $x$ is the real part of $z$ and that $y$ is its imaginary part. For two subsets $X, Y \subseteq V$, we write

$$
X+i Y=\left\{(x+i y) \in V_{\mathbf{C}} \mid x \in X \text { and } y \in Y\right\} .
$$

Let $H$ be a hyperplane of $V$. The complexification $H_{\mathbf{C}}$ of $H$ is the hyperplane of $V_{\mathbf{C}}$ spanned by $H ; H_{\mathbf{C}}=H+i H$.

Let $\mathscr{A}$ be an arrangement of hyperplanes in a real vector space $V$. We set

$$
M(\mathscr{A})=V_{\mathbf{C}}-\left(\bigcup_{H \in \mathscr{A}} H_{\mathbf{C}}\right)
$$

This space is an open and connected submanifold of $V_{\mathbf{C}}$. We say that $\mathscr{A}$ is a $K(\pi, 1)$ arrangement if $M(\mathscr{A})$ is a $K(\pi, 1)$ space.

The lattice of a real arrangement $\mathscr{A}$ of hyperplanes is the poset

$$
\mathscr{L}(\mathscr{A})=\left\{\bigcap_{H \in \mathscr{B}} H \mid \mathscr{B} \subseteq \mathscr{A}\right\}
$$

ordered by the reverse inclusion. $V=\cap_{H \in \emptyset} H$ is the smallest element of $\mathscr{L}(\mathscr{A})$, and $\cap_{H \in \mathscr{A}} H$ is the greatest one. For $X \in \mathscr{L}(\mathscr{A})$, we set

$$
\mathscr{A}_{X}=\{H \in \mathscr{A} \mid H \supseteq X\} .
$$

Let $\mathscr{A}$ be a real and essential arrangement of hyperplanes. A chamber of $\mathscr{A}$ is a connected component of $V-\cup_{H \in \mathscr{A}} H$. We say that $\mathscr{A}$ is simplicial if every

Received October 21, 1991. 
chamber of $\mathscr{A}$ is an open simplicial cone. In [De], for a simplicial arrangement $\mathscr{A}$ of hyperplanes, Deligne constructs a cover $q: \hat{M}(\mathscr{A}) \rightarrow M(\mathscr{A})$, defines a simplicial complex $\operatorname{Del}(\mathscr{A})$ from $\mathscr{A}$, and proves that $\operatorname{Del}(\mathscr{A})$ has the same homotopy type as $\hat{M}(\mathscr{A})$, and that $\operatorname{Del}(\mathscr{A})$ is contractible. In particular, $q: \hat{M}(\mathscr{A}) \rightarrow M(\mathscr{A})$ is the universal cover of $M(\mathscr{A})$, and $\mathscr{A}$ is a $K(\pi, 1)$ arrangement.

In [Pa1], the author generalizes Deligne's construction of the universal cover $q: \hat{M}(\mathscr{A}) \rightarrow M(\mathscr{A})$ of $M(\mathscr{A})$ to any real arrangement $\mathscr{A}$ of hyperplanes using a new combinatorial tool: the oriented systems.

Our goal in this paper is to generalize the defintion of the Deligne complex $\operatorname{Del}(\mathscr{A})$ to any real and essential arrangement $\mathscr{A}$ of hyperplanes (in the general case, $\operatorname{Del}(\mathscr{A})$ is a regular and normal CW-complex), and to prove the following result.

Main Theorem. Let $\mathscr{A}$ be a real and essential arrangement of hyperplanes. The Deligne complex $\operatorname{Del}(\mathscr{A})$ of $\mathscr{A}$ has the same homotopy type as the universal cover $\hat{M}(\mathscr{A})$ of $M(\mathscr{A})$ if and only if $\mathscr{A}_{X}$ is a $K(\pi, 1)$ arrangement for every $X \in \mathscr{L}(\mathscr{A})$ different from $\{0\}$.

In particular, if $\mathscr{A}$ is an essential arrangement of hyperplanes in a real vector space of dimension $\leq 3$, then $\operatorname{Del}(\mathscr{A})$ has the same homotopy type as the universal cover $\hat{M}(\mathscr{A})$ of $M(\mathscr{A})$ (it is well known that any arrangement of hyperplanes in a real vector space of dimension $\leq 2$ is a $K(\pi, 1)$ arrangement).

Note that the study of the topology of $M(\mathscr{A})$, where $\mathscr{A}$ is an arbitrary real arrangement of hyperplanes, can be easily reduced to the case of an essential arrangement. Thus the hypothesis " $\mathscr{A}$ is essential" is not a restriction.

At the end of this section we will prove that: "if $\mathscr{A}$ is a $K(\pi, 1)$ arrangement, then $\mathscr{A}_{X}$ is also a $K(\pi, 1)$ arrangement for every $X \in \mathscr{L}(\mathscr{A})$ " (Lemma 1.1). It follows that, if $\mathscr{A}$ is a $K(\pi, 1)$ arrangement, then $\operatorname{Del}(\mathscr{A})$ has the same homotopy type as the universal cover $\hat{M}(\mathscr{A})$ of $M(\mathscr{A})$, and, consequently, $\operatorname{Del}(\mathscr{A})$ is contractible. In view of these facts, our complex $\operatorname{Del}(\mathscr{A})$ can certainly be used to prove that a given real arrangement of hyperplanes is a $K(\pi, 1)$ arrangement.

We refer to [FR] for a good exposition on $K(\pi, 1)$ arrangements, and to [Or] and [OT] for good expositions on the theory of arrangements of hyperplanes.

Our work is organized as follows.

Section 2 is a summary of [Pa1]. Its aim is to introduce our main combinatorial tool, the oriented systems, and to give the construction of the universal cover 
$q: \hat{M}(\mathscr{A}) \rightarrow M(\mathscr{A})$ of $M(\mathscr{A})$. Although this section is almost identical to Section 2 of $[\mathrm{Pa} 2]$, for convenience we reproduce it here rather than referring the reader to the original paper.

In Section 3, we define the complex $\operatorname{Del}(\mathscr{A})$ and prove the Main Theorem.

I am grateful to Peter Orlik and Hiroaki Terao who have helped me with discussions, suggestions and encouragement during my work. I am also grateful to Mutsuo Oka for granting his permission to include in this paper his proof of Lemma 1.1.

Lemma 1.1. Let $\mathscr{A}$ be a real arrangement of hyperplanes, and let $X \in \mathscr{L}(\mathscr{A})$. If $\mathscr{A}$ is a $K(\pi, 1)$ arrangement, then $\mathscr{A}_{X}$ is also a $K(\pi, 1)$ arrangement.

Proof. Let $\iota^{1}: M(\mathscr{A}) \rightarrow M\left(\mathscr{A}_{X}\right)$ be the inclusion map of $M(\mathscr{A})$ into $M\left(\mathscr{A}_{X}\right)$. We are going to prove that $\iota^{1}$ admits a right homotopy inverse. This shows that $\left(\iota^{1}\right)_{*}: \pi_{n}(M(\mathscr{A})) \rightarrow \pi_{n}\left(M\left(\mathscr{A}_{X}\right)\right)$ is a surjective morphism of groups for every $n \geq 0$, and thus that $M\left(\mathscr{A}_{X}\right)$ is a $K(\pi, 1)$ space if $M(\mathscr{A})$ is a $K(\pi, 1)$ space.

Pick a point $z \in \cap_{H \in \mathscr{A}_{X}} H_{\mathbf{C}}$ such that $z \notin H_{\mathbf{C}}$ for any $H \in \mathscr{A}_{-}-\mathscr{A}_{X}$. Choose a small disk $\mathbf{B}$ in $V_{\mathbf{C}}$ centered in $z$ and which does not intersect any hyperplane $H_{\mathbf{C}}$ with $H \in \mathscr{A}-\mathscr{A}_{X}$. Set

$$
W=\mathbf{B}-\left(\bigcup_{H \in \mathscr{A}_{X}} H_{\mathbf{C}}\right)=\mathbf{B}-\left(\bigcup_{H \in \mathscr{A}} H_{\mathbf{C}}\right)
$$

and let $\iota^{0}: W \rightarrow M(\mathscr{A})$ denote the inclusion map of $W$ into $M(\mathscr{A})$. Then $\iota=\iota^{1} \circ \iota^{0}$ $: W \rightarrow M\left(\mathscr{A}_{X}\right)$ is obviously a homotopy equivalence, thus $\iota^{1}$ admits a right homotopy inverse.

Note that Lemma 1.1 can be easily generalized to complex arrangements of hyperplanes.

\section{The universal cover of $M(\mathscr{A})$}

This section is divided into three subsections. In the first one we introduce our main combinatorial tool: the oriented systems. In the second subsection we define the oriented system $(\Gamma(\mathscr{A}), \sim)$ associated with a real arrangement $\mathscr{A}$ of hyperplanes. In the third subsection, using the universal cover $\rho:(\hat{\Gamma}(\mathscr{A}), \sim) \rightarrow$ $(\Gamma(\mathscr{A}), \sim)$ of the oriented system $(\Gamma(\mathscr{A}), \sim)$, we give the construction of the universal cover $q: \hat{M}(\mathscr{A}) \rightarrow M(\mathscr{A})$ of $M(\mathscr{A})$. 
All results stated in this section are derived from [Pa1], so we will not give any proofs.

\section{A. Oriented systems}

An oriented graph $\Gamma$ is the following data:

$1)$ a set $V(\Gamma)$ of vertices,

2) a subset $A(\Gamma) \subseteq(V(\Gamma) \times V(\Gamma))-\{(v, v) \mid v \in V(\Gamma)\}$ of arrows.

The origin of an arrow $a=(v, w)$ is $v$ and its end is $w$. An oriented graph $\Gamma$ is locally finite if every vertex $v \in V(\Gamma)$ is the origin or the end of only a finite number of arrows.

A path of an oriented graph $\Gamma$ is an expression

$$
f=a_{1}^{\varepsilon_{1}} a_{2}^{\varepsilon_{2}} \cdots a_{n}^{\varepsilon_{n}}
$$

where $a_{i} \in A(\Gamma)$ and $\varepsilon_{i} \in\{ \pm 1\}$ (for $i=1, \ldots, n$ ), such that there exists a sequence $v_{0}, v_{1}, \ldots, v_{n}$ of vertices of $\Gamma$ with:

$$
\begin{aligned}
& a_{i}=\left(v_{i-1}, v_{i}\right) \text { if } \varepsilon_{i}=1 \text { and } \\
& a_{i}=\left(v_{i}, v_{i-1}\right) \text { if } \varepsilon_{i}=-1 .
\end{aligned}
$$

We say that $v_{0}$ is the origin of $f$ and that $v_{n}$ is its end. The integer $n$ is its length and $\sum_{i=1}^{n} \varepsilon_{i}$ is its weight. Every vertex of $\Gamma$ is assumed to be a path of length 0 and of weight 0 . For a path $f=a_{1}^{\varepsilon_{1}} \cdots a_{n}^{\varepsilon_{n}}$, we write $f^{-1}=a_{n}^{-\varepsilon_{n}} \cdots a_{1}^{-\varepsilon_{1}}$. For two paths $f=a_{1}^{\varepsilon_{1}} \cdots a_{n}^{\varepsilon_{n}}$ and $g=b_{1}^{\mu_{1}} \cdots b_{m}^{\mu_{m}}$ with end $(f)=\operatorname{origin}(g)$, we write $f g$ $=a_{1}^{\varepsilon_{1}} \cdots a_{n}^{\varepsilon_{n}} b_{1}^{\mu_{1}} \cdots b_{m}^{\mu_{m}}$.

An oriented graph $\Gamma$ is connected if, for every pair $(v, w)$ of vertices of $\Gamma$, there exists a path of $\Gamma$ which begins at $v$ and ends in $w$.

We always assume the oriented graphs to be locally finite and connected.

Let $\Gamma$ be an oriented graph. An identification of $\Gamma$ is an equivalence relation $\sim$ in the set of paths of $\Gamma$ with the following properties:

1) $f \sim g \Rightarrow \operatorname{origin}(f)=\operatorname{origin}(g)$, end $(f)=\operatorname{end}(g)$ and weight $(f)=$ weight $(g)$,

2) $f f^{-1} \sim \operatorname{origin}(f)$, for every path $f$,

3) $f \sim \mathrm{g} \Rightarrow f^{-1} \sim g^{-1}$,

4) $f \sim g \Rightarrow h_{1} f h_{2} \sim h_{1} g h_{2}$, for suitable paths $h_{1}$ and $h_{2}$.

An oriented system is a pair $(\Gamma, \sim)$, where $\Gamma$ is an oriented graph and $\sim$ is an identification of $\Gamma$. 
Let $\rho: \Theta \rightarrow \Gamma$ be a morphism of oriented graphs. We say that $\rho$ is a cover of $\Gamma$ if, for every vertex $v$ of $\Theta$ and every path $f$ of $\Gamma$ beginning at $\rho(v)$, there exists a unique path $\hat{f}$ of $\Theta$ such that $\operatorname{origin}(\hat{f})=v$ and $\rho(\hat{f})=f$.

Let $\rho:(\Theta, \sim) \rightarrow(\Gamma, \sim)$ be a morphism of oriented systems (i.e. $\hat{f} \sim \hat{g} \Rightarrow$ $\rho(\hat{f}) \sim \rho(\hat{g}))$. We say that $\rho$ is a cover of $(\Gamma, \sim)$ if it has the following two properties.

1) $\rho: \Theta \rightarrow \Gamma$ is a cover of $\Gamma$.

2) Let $v \in V(\Theta)$, let $f$ and $g$ be two paths of $\Gamma$ which both begin at $\rho(v)$, and let $\hat{f}$ and $\hat{g}$ be the lifts of $f$ and $g$ respectively into $\Theta$ beginning at $v$. If $f \sim g(\Rightarrow$ $\operatorname{end}(f)=\operatorname{end}(g))$, then $\hat{f} \sim \hat{g}(\Leftrightarrow \operatorname{end}(\hat{f})=\operatorname{end}(\hat{g}))$.

PROPOSITION 2.1. Let $(\Gamma, \sim)$ be an oriented system. There exists a unique cover $\pi:(\hat{\Gamma}, \sim) \rightarrow(\Gamma, \sim)$ of $(\Gamma, \sim)$ (up to isomorphism) which has the following universal property.

If $\rho:(\Theta, \sim) \rightarrow(\Gamma, \sim)$ is a cover of $(\Gamma, \sim)$, then there exists a unique cover $\pi^{\prime}:(\Gamma, \dot{\sim}) \rightarrow(\Theta, \sim)$ of $(\Theta, \sim)$ (up to isomorphism) such that $\pi=\rho^{\circ} \pi^{\prime}$.

We call $\pi:(\hat{\Gamma}, \sim) \rightarrow(\Gamma, \sim)$ the universal cover of $(\Gamma, \sim)$.

Proposition 2.2. Let $\pi:(\hat{\Gamma}, \sim) \rightarrow(\Gamma, \sim)$ be the universal cover of an oriented system $(\Gamma, \sim)$. Two paths $\hat{f}$ and $\hat{g}$ of $\hat{\Gamma}$ are identified by $\sim$ if and only if $\operatorname{origin}(\hat{f})=\operatorname{origin}(\hat{g})$ and end $(\hat{f})=\operatorname{end}(\hat{g})$.

\section{B. Definition of $(\Gamma(\mathscr{A}), \sim)$}

Let $\mathscr{A}$ be an arrangement of hyperplanes in a real vector space $V$. The hyperplanes of $\mathscr{A}$ subdivide $V$ into facets. We denote by $\mathscr{F}(\mathscr{A})$ the set of all the facets. The support $|F|$ of a facet $F$ is the vector space $|F| \in \mathscr{L}(\mathscr{A})$ spanned by $F$. Every facet is open in its support. We denote by $\bar{F}$ the closure of $F$ in $V$. There is a partial order in $\mathscr{F}(\mathscr{A})$ defined by $F \leq G$ if $F \subseteq \bar{G}$.

A chamber of $\mathscr{A}$ is a facet of codimension 0 . A face is a facet of codimension 1 . Two chambers $C$ and $D$ are adjacent if they have a common face (i.e. a common facet of codimension 1).

Now, let us define the oriented system $(\Gamma(\mathscr{A}), \sim)$ associated with $\mathscr{A}$.

The vertices of $\Gamma(\mathscr{A})$ are the chambers of $\mathscr{A}$. An arrow of $\Gamma(\mathscr{A})$ is a pair $(C, D)$, where $C$ and $D$ are adjacent chambers. Note that, in this oriented graph, if $(C, D)$ is an arrow, then $(D, C)$ is also an arrow. 
A positive path of an oriented graph $\Delta$ is a path $f=a_{1}^{\varepsilon_{1}} \cdots a_{n}^{\varepsilon_{n}}$ with $\varepsilon_{1}=\ldots$ $=\varepsilon_{n}=1$. This positive path is minimal if there is no positive path in $\Delta$ having the same origin as $f$, the same end as $f$, and a length smaller than the one of $f$.

The relation $\sim$ is the smallest identification of $\Gamma(\mathscr{A})$ such that:

if $f$ and $g$ are both positive minimal paths with the same origin and the same end, then $f \sim g$.

\section{C. Universal cover of $M(\mathscr{A})$}

Let $\mathscr{A}$ be an arrangement of hyperplanes in a real vector space $V$. We set

$$
M(\mathscr{A})=V_{\mathbf{C}}-\left(\bigcup_{H \in \mathscr{A}} H_{\mathbf{C}}\right)
$$

Our goal in this subsection is to explain the construction of the universal cover $q: \hat{M}(\mathscr{A}) \rightarrow M(\mathscr{A})$ of $M(\mathscr{A})$.

Let $C$ be a chamber of $\mathscr{A}$. For a facet $F \in \mathscr{F}(\mathscr{A})$, we denote by $C_{F}$ the unique chamber of $\mathscr{A}_{|F|}$ containing $C$. We write

$$
\left.M(C)=\bigcup_{F \in \mathscr{F}(\mathscr{A})}\left(F+i C_{F}\right) \subseteq V+i V\right)=V_{\mathbf{C}}
$$

Note that this union is disjoint.

Lemma 2.3. The set $\{M(C) \mid C \in V(\Gamma(\mathscr{A}))\}$ is a covering of $M(\mathscr{A})$ by open subsets.

Now, consider the universal cover $\rho:(\hat{\Gamma}(\mathscr{A}), \sim) \rightarrow(\Gamma(\mathscr{A}), \sim)$ of $(\Gamma(\mathscr{A}), \sim)$. For every vertex $v$ of $\hat{\Gamma}(\mathscr{A})$, write

$$
M(v)=M(\rho(v)) .
$$

Set

$$
M^{\prime}(\mathscr{A})=\amalg_{v \in V(\hat{\Gamma}(\mathscr{A}))} M(v)
$$

and let

$$
q^{\prime}: M^{\prime}(\mathscr{A}) \rightarrow M(\mathscr{A})
$$

be the natural projection. 
It is easy to see that, if two chambers $C$ and $D$ are adjacent, then there is only one hyperplane $H \in \mathscr{A}$ which separates $C$ and $D$; it is the support of their common face. For a chamber $C$ of $\mathscr{A}$ and a hyperplane $H \in \mathscr{A}$, we denote by $H_{C}^{+}$ the open half-space of $V$ bordered by $H$ and containing $C$.

Let $R$ be the smallest equivalence relation on $M^{\prime}(\mathscr{A})$ such that:

if $a=(v, w) \in \mathscr{A}(\hat{\Gamma}(\mathscr{A})), z \in M(v), z^{\prime} \in M(w)$, and

$$
q^{\prime}(z)=q^{\prime}\left(z^{\prime}\right) \in M(v) \cap M(w) \cap\left(H_{\rho(w)}^{+}+i V\right),
$$

where $H$ is the unique hyperplane of $\mathscr{A}$ which separates $\rho(v)$ and $\rho(w)$, then

$$
z \mathscr{R} z^{\prime} .
$$

The space $\hat{M}(\mathscr{A})$ is the quotient

$$
\hat{M}(\mathscr{A})=M^{\prime}(\mathscr{A}) / \mathscr{R},
$$

and

$$
q: \hat{M}(\mathscr{A}) \rightarrow M(\mathscr{A})
$$

is the map induced by $q^{\prime}$.

THEOREM 2.4. The map $q: \hat{M}(\mathscr{A}) \rightarrow M(\mathscr{A})$ is the universal cover of $M(\mathscr{A})$.

The following Lemmas $2.5,2.6$ and 2.7 are in [Pa1] preliminary results to the proof of Theorem 2.4; nevertheless, we state them since they will be used later in this paper.

Fix a vertex $v \in V(\hat{\Gamma}(\mathscr{A}))$. Write $C=\rho(v)$. For every chamber $D$ of $\mathscr{A}$, we choose a positive minimal path $f_{D}$ of $\Gamma(\mathscr{A})$ beginning at $C$ and ending in $D$. We denote by $\hat{f}_{D}$ the lift of $f_{D}$ into $\hat{\Gamma}(\mathscr{A})$ beginning at $v$. Note that the end of $\hat{f}_{D}$ does not depend on the choice of $f_{D}$ (see the definition of the identification $\sim$ of $\Gamma(\mathscr{A})$ ). We set

$$
\sum(v)=\left\{\operatorname{end}\left(\hat{f}_{D}\right) \mid D \in V(\Gamma(\mathscr{A}))\right\} .
$$

The restriction of $\rho$ to $\sum(v)$ is clearly a bijection $\sum(v) \rightarrow V(\Gamma(\mathscr{A}))$.

Let $v$ and $w$ be two vertices of $\hat{\Gamma}(\mathscr{A})$. We write

$$
\bar{Z}(v, w)=\bigcup_{u} \bar{\rho}(u),
$$

where the union is over all vertices $u \in \sum(v) \cap \sum(w)$ and, for $u \in \sum(v)$ $\cap \sum(w)$, the set $\bar{\rho}(u)$ is the closure of $\rho(u)$ in $V$. We denote by $Z(v, w)$ the in- 
terior of $\bar{Z}(v, w)$. Note that $Z(v, w)$ is a union of facets of $\mathscr{A}$.

Consider the natural projection

$$
p: M^{\prime}(\mathscr{A})=\underset{v \in V(\hat{\Gamma}(\mathscr{A}))}{\amalg} M(v) \rightarrow \hat{M}(\mathscr{A}) .
$$

For every $v \in V(\hat{\Gamma}(\mathscr{A}))$, we write $\hat{M}(v)=p(M(v))$. Since $q^{\prime}: M^{\prime}(\mathscr{A}) \rightarrow M(\mathscr{A})$ sends $M(v)$ homeomorphically onto $M(v)$, and $q^{\prime}: q \circ p$, the map $q: \hat{M}(\mathscr{A}) \rightarrow$ $M(\mathscr{A})$ sends $\hat{M}(v)$ homeomorphically onto $M(v)$. Moreover, since $q$ is a cover, $\hat{M}(v)$ is an open subset of $\hat{M}(\mathscr{A})$.

Lemma 2.5. Let $v$ and $w$ be two vertices of $\hat{\Gamma}(\mathscr{A})$. The border of $Z(v, w)$ is contained in the union of the hyperplanes $H \in \mathscr{A}$ which separate $\rho(v)$ and $\rho(w)$.

Lemma 2.6. Let $v$ and $w$ be two vertices of $\hat{\Gamma}(\mathscr{A})$. Then

$$
q(\hat{M}(v) \cap \hat{M}(w))=M(v) \cap M(w) \cap(Z(v, w)+i V) .
$$

Corollary. Let $v, w$ be two vertices of $\hat{\Gamma}(\mathscr{A})$. If $\sum(v) \cap \sum(w)=\emptyset$, then $\hat{M}(v) \cap M(w)=\emptyset$.

Lemma 2.7. For every chamber $C$ of $\mathscr{A}$, we have

$$
q^{-1}(M(C))=\bigcup_{v \in \rho^{-1}(C)} \hat{M}(v)
$$

and this union is disjoint.

\section{The Deligne complex of $\mathscr{A}$}

Throughout this section, $\mathscr{A}$ is an essential arrangement of hyperplanes in a real vector space $V$ of dimension $l$, the map $q: \hat{M}(\mathscr{A}) \rightarrow M(\mathscr{A})$ is the universal cover of $M(\mathscr{A})$, the pair $(\Gamma(\mathscr{A}), \sim)$ is the oriented system associated with $\mathscr{A}$, and $\rho:(\hat{\Gamma}(\mathscr{A}), \sim) \rightarrow(\Gamma(\mathscr{A}), \sim)$ is the universal cover of $(\Gamma(\mathscr{A}), \sim)$.

We provide $V$ with an arbitrary scalar product. Let $\mathbf{S}^{l-1}=\{x \in V \mid\|x\|=1\}$ be the unit sphere. The arrangement $\mathscr{A}$ determines a cellular decomposition of $\mathbf{S}^{l-1}$. With a facet $F$ of $\mathscr{A}$ of dimension $d$ corresponds the (closed) cell $\Delta_{d-1}(F)=$ $\bar{F} \cap \mathbf{S}^{l-1}$ of dimension $(d-1)$, and every cell of this decomposition has that form.

For every vertex $v$ of $\hat{\Gamma}(\mathscr{A})$, we write 


$$
\Delta_{l-1}^{\prime}(v)=\Delta_{l-1}(\rho(v))
$$

(recall that $\rho(v)$ is a chamber of $\mathscr{A}$, so is a facet of dimension $l$ ). We set

$$
\operatorname{Del}^{\prime}(\mathscr{A})=\underset{v}{\amalg} \Delta_{l-1}^{\prime}(v),
$$

where the union is over all the vertices $v$ of $\hat{\Gamma}(\mathscr{A})$, and let

$$
\pi^{\prime}: \operatorname{Del}^{\prime}(\mathscr{A}) \rightarrow \mathbf{S}^{l-1}
$$

be the natural projection, The space $\operatorname{Del}^{\prime}(\mathscr{A})$ is a disjoint union of $(l-1)$-cells, and each cell $\Delta_{l-1}^{\prime}(v)$ has a natural cellular decomposition given by the embedding $\Delta_{l-1}^{\prime}(v) \hookrightarrow \mathbf{S}^{l-1}$. Thus $\operatorname{Del}^{\prime}(\mathscr{A})$ can be viewed as a cellular complex, and $\pi^{\prime}$ as a cellular map.

Let $\mathscr{R}$ be the smallest equivalence relation on $\operatorname{Del}^{\prime}(\mathscr{A})$ such that:

if $a=(v, w) \in A(\Gamma(\mathscr{A})), \alpha \in \Delta_{l-1}^{\prime}(v), \beta \in \Delta_{l-1}^{\prime}(w)$, and $\pi^{\prime}(\alpha)=\pi^{\prime}(\beta)$, then

$$
\alpha \mathscr{R} \beta .
$$

We denote by $\operatorname{Del}^{0}(\mathscr{A})$ the quotient

$$
\operatorname{Del}^{\circ}(\mathscr{A})=\operatorname{Del}(\mathscr{A}) / \mathscr{R},
$$

by

$$
\tau: \operatorname{Del}^{\prime}(\mathscr{A}) \rightarrow \operatorname{Del}^{\circ}(\not A)
$$

the natural projection, and by

$$
\pi^{o}: \operatorname{Del}^{o}(\mathscr{A}) \rightarrow \mathbf{S}^{l-1}
$$

the map induced by $\pi^{\prime}$. In other words, The space $\operatorname{Del}^{o}(\mathscr{A})$ is obtained from $\operatorname{Del}^{\prime}(\mathscr{A})$ as follows: for every arrow $a=(v, w)$ of $\hat{\Gamma}(\mathscr{A})$, we identify the $(l-2)$-cell $\Delta_{l-1}(F) \subset \Delta_{l-1}^{\prime}(v)$ with the $(l-2)$-cell $\Delta_{l-2}(F) \subseteq \Delta_{l-1}^{\prime}(w)$, where $F$ is the face of $\mathscr{A}$ common to $\rho(v)$ and $\rho(w)$. Thus $\operatorname{Del}^{o}(\mathscr{A})$ has a natural cellular decomposition where the maps $\tau$ and $\pi^{\circ}$ are cellular maps.

For every vertex $v$ of $\hat{\Gamma}(\mathscr{A})$, we write $\Delta_{l-1}^{o}(v)=\tau\left(\Delta_{l-1}^{\prime}(v)\right)$.

For every vertex $v$ of $\hat{\Gamma}(\mathscr{A})$, we write

$$
\mathbf{S}^{l-1}(v)=\bigcup_{u \in \Sigma(v)} \Delta_{l-1}^{o}(u) \subseteq \operatorname{Del}^{o}(\mathscr{A})
$$

(the definition of $\Sigma(v)$ is given in Subsection 3.C). The restriction of $\pi^{o}$ to $\mathbf{S}^{l-1}(v)$ is obviously an isomorphism $\mathbf{S}^{l-1}(v) \rightarrow \mathbf{S}^{l-1}$ of cellular complexes. 
The Deligne complex of $\mathscr{A}$ is the cellular complex $\operatorname{Del}(\mathscr{A})$ obtained from $\operatorname{Del}^{o}(\mathscr{A})$ by attaching a $l$-cell $\mathbf{B}^{l}(v)$ to $\operatorname{Del}^{o}(\mathscr{A})$ having $\mathbf{S}^{l-1}(v)$ as border, for every vertex $v$ of $\hat{\Gamma}(\mathscr{A})$.

The complexes $\mathbf{S}^{l-1}, \operatorname{Del}^{o}(\mathscr{A})$ and $\operatorname{Del}(\mathscr{A})$ are clearly regular and normal CW-complexes.

MAIN THEOREm. Let $\mathscr{A}$ be a real and essential arrangement of hyperplanes. The Deligne complex $\operatorname{Del}(\mathscr{A})$ of $\mathscr{A}$ has the same homotopy type as the universal cover $\hat{M}(\mathscr{A})$ of $M(\mathscr{A})$ if and only if $\mathscr{A}_{X}$ is a $K(\pi, 1)$ arrangement for every $X \in$ $\mathscr{L}(\mathscr{A})$ different from $\{0\}$.

COROllary 1. Let $\mathscr{A}$ be an essential arrangement of hyperplanes in a real vector space $V$ of dimension $\leq 3$. Then $\operatorname{Del}(\mathscr{A})$ has the same homotopy type as the universal cover $\hat{M}(\mathscr{A})$ of $M(\mathscr{A})$.

Corollary 2. Let $\mathscr{A}$ be a real, essential, and $K(\pi, 1)$ arrangement of hyperplanes. Then $\operatorname{Del}(\mathscr{A})$ has the same homotopy type as the universal cover $\hat{M}(\mathscr{A})$ of $M(\mathscr{A})$. In particular, $\operatorname{Del}(\mathscr{A})$ is contractible.

Let $N$ be a regular and normal $\mathrm{CW}$-complex. The cellular decomposition of $N$ determines a simplicial decomposition of $N$ called the barycentric subdivision of $N$ (see [LW, Ch. III, Theorem 1.7]). For every cell $\Delta_{d}$ of $N$ we fix a point $w\left(\Delta_{d}\right) \in$ $\left(\Delta_{d}-\partial \Delta_{d}\right)$, where $\partial \Delta_{d}$ is the border of $\Delta_{d}$ (we assume $\partial \Delta_{d}=\emptyset$ if $\operatorname{dim}\left(\Delta_{d}\right)=0$ ). A chain $\Delta_{d_{0}} \subset \Delta_{d_{1}} \subset \ldots \subset \Delta_{d_{r}}$ of cells of $N$ determines a simplex $\Phi=\omega\left(\Delta_{d_{0}}\right) \vee$ $\omega\left(\Delta_{d_{1}}\right) \vee \ldots \vee \omega\left(\Delta_{d_{r}}\right)$ having $\omega\left(\Delta_{d_{0}}\right), \omega\left(\Delta_{d_{1}}\right), \ldots, \omega\left(\Delta_{d_{r}}\right)$ as vertices and included in $\left(\Delta_{d_{r}}-\partial \Delta_{d_{r}}\right)$, and every simplex of this simplicial decomposition has that form. All the simplexes are assumed to be open.

From now on, we assume $\mathbf{S}^{l-1}, \operatorname{Del}^{o}(\mathscr{A})$ and $\operatorname{Del}(\mathscr{A})$ to be provided with their respective barycentric subdivisions; moreover, we assume all the simplexes of $\mathbf{S}^{l-1}$ to be convex subsets of $\mathbf{S}^{l-1}$, the complex $\operatorname{Del}^{0}(\mathscr{A})$ to be a simplicial subcomplex of $\operatorname{Del}(\mathscr{A})$, and $\pi^{o}: \operatorname{Del}^{o}(\mathscr{A}) \rightarrow \mathbf{S}^{l-1}$ to be a simplicial map.

Notations. Let $\phi$ be a simplex of $\mathbf{S}^{l-1}$. Then, by the construction of the barycentric subdivision of $\mathbf{S}^{l-1}$, the simplex $\phi$ is contained in a unique facet of $\mathscr{A}$ which we denote by $F(\phi)$. We write $X(\phi)=|F(\phi)|$. Note that $X(\phi) \neq\{0\}$.

For a simplex $\Phi^{o}$ of $\operatorname{Del}^{o}(\mathscr{A})$, we write $F\left(\Phi^{o}\right)=F\left(\pi^{o}\left(\Phi^{o}\right)\right)$ and $X\left(\Phi^{o}\right)=$ $X\left(\pi^{o}\left(\Phi^{o}\right)\right)$. 
The proof of the Main Theorem is divided in 5 parts.

In Part 1, we give some preliminary results on the oriented system associated with $\mathscr{A}$.

In Part 2, to every simplex $\Phi$ of $\operatorname{Del}(\mathscr{A})$ we associate a nonempty open subset $U(\Phi)$ of $\hat{M}(\mathscr{A})$.

In Part 3, we prove the following assertions.

1) Let $\omega_{0}, \omega_{1}, \ldots, \omega_{r}$ be $(r+1)$ vertices of $\operatorname{Del}(\mathscr{A})$. If $\cap_{i=0}^{r} U\left(\omega_{i}\right) \neq \emptyset$, then $\omega_{0}, \omega_{1}, \ldots, \omega_{r}$ are the vertices of a simplex $\Phi$ of $\operatorname{Del}(\mathscr{A})$.

2) Let $\omega_{0}, \omega_{1}, \ldots, \omega_{r}$ be the vertices of a simplex $\Phi$ of $\operatorname{Del}(\mathscr{A})$. Then $\cap_{i=0}^{r} U\left(\omega_{\imath}\right)=U(\Phi)$.

3) The set $U=\{U(\omega) \mid \omega$ a vertex of $\operatorname{Del}(\mathscr{A})\}$ is a covering of $\hat{M}(\mathscr{A})$.

Assertions 1), 2) and 3) show that $\mathcal{U}=\{U(\omega) \mid \omega$ a vertex of $\operatorname{Del}(\mathscr{A})\}$ is a covering of $\hat{M}(\mathscr{A})$ having $\operatorname{Del}(\mathscr{A})$ as nerve.

In Part 4 , we prove the following assertions.

1) Let $v$ be a vertex of $\hat{\Gamma}(\mathscr{A})$. Then $U\left(\omega\left(\mathbf{B}^{l}(v)\right)\right)$ is contractible.

2) Let $v$ be a vertex of $\hat{\Gamma}(\mathscr{A})$, and let $\Phi^{\circ}$ be a simplex of $\operatorname{Del}^{\circ}(\mathscr{A})$ contained in $\mathbf{S}^{l-1}(v)$. Write $\Phi=\Phi^{o} \vee \omega\left(\mathbf{B}^{l}(v)\right)$. Then $U(\Phi)$ is contractible.

3) Let $\Phi^{o}$ be a simplex of $\operatorname{Del}^{\circ}(\mathscr{A})$. Then $U\left(\Phi^{\circ}\right)$ has the same homotopy type as the universal cover $\hat{M}\left(\mathscr{A}_{X\left(\Phi^{\circ}\right)}\right)$ of $M\left(\mathscr{A}_{X\left(\Phi^{\circ}\right)}\right)$.

In particular, if $\mathscr{A}_{X}$ is a $K(\pi, 1)$ arrangement for every $X \in \mathscr{L}(\mathscr{A})$ different from $\{0\}$, then $U\left(\Phi^{\circ}\right)$ is contractible for every simplex $\Phi^{\circ}$ of $\operatorname{Del}^{\circ}(\mathscr{A})$ (since $U\left(\Phi^{\circ}\right)$ has the same homotopy type as $\hat{M}\left(\mathscr{A}_{X\left(\Phi^{\circ}\right)}\right)$ and $\left.X\left(\Phi^{\circ}\right) \neq\{0\}\right)$. This fact, Assertion 2) of Part 3, and Assertions 1) and 2) of Part 4 show that every nonempty intersection of elements of $\mathcal{U}$ is contractible, thus, by [We], $\operatorname{Del}(\mathscr{A})$ has the same homotopy type as $\hat{M}(\mathscr{A})$ (since $\mathcal{U}$ is a covering of $\hat{M}(\mathscr{A})$ having $\operatorname{Del}(\mathscr{A})$ as nerve).

In Part 5, we assume that there exists an $X \in \mathscr{L}(\mathscr{A})$ different from $\{0\}$ such that $\mathscr{A}_{X}$ is not a $K(\pi, 1)$ arrangement. Then we construct a new space $\hat{M}_{\infty}$ by attaching cells to $\hat{M}(\mathscr{A})$ such that:

a) $\operatorname{Del}(\mathscr{A})$ has the same homotopy type as $\hat{M}_{\infty}$,

b) there exists an integer $n_{0}>0$ such that $\pi_{n_{0}}(\hat{M}(\mathscr{A})) \neq \pi_{n_{0}}\left(\hat{M}_{\infty}\right)$.

\section{Part 1.}

Let $\Gamma$ be an oriented graph, and let $W$ be a subset of $V(\Gamma)$. The oriented subgraph of $\Gamma$ generated by $W$ is the oriented graph $\Theta$ having $W$ as set of vertices and $\{(v, w) \in A(\Gamma) \mid v, w \in W\}$ as set of arrows.

For a facet $F$ of $\mathscr{A}$, we denote by $\Gamma_{F}$ the oriented subgraph of $\Gamma(\mathscr{A})$ 
generated by $\{C \in V(\Gamma(\mathscr{A})) \mid C$ has $F$ as facet $\}$. For a simplex $\Phi^{o}$ of $\operatorname{Del}^{\circ}(\mathscr{A})$, we denote by $\hat{\Gamma}_{\Phi^{o}}$ the oriented subgraph of $\hat{\Gamma}(\mathscr{A})$ generated by $\{v \in V(\hat{\Gamma}(\mathscr{A})) \mid$ $\left.\Delta_{l-1}^{o}(v) \supseteq \Phi^{o}\right\}$.

A gallery of $\mathscr{A}$ is a sequence $\left(C_{0}, C_{1}, \ldots, C_{n}\right)$ of chambers of $\mathscr{A}$ such that $C_{i-1}$ and $C_{i}$ are adjacent for $i=1, \ldots, n$ (here we assume $C_{i-1} \neq C_{i}$ ). Any positive path $f=a_{1} \ldots a_{n}$ of $\Gamma(\mathscr{A})$ can be viewed as the gallery $G=\left(C_{0}, C_{1}, \ldots, C_{n}\right)$, where $C_{i}=\operatorname{end}\left(a_{1}, \ldots, a_{i}\right)$ for $i=0,1, \ldots, n$. In particular, if $f=a_{1} \ldots a_{n}$ is a positive minimal path of $\Gamma(\mathscr{A})$ then $G=\left(C_{0}, C_{1}, \ldots, C_{n}\right)$ is a minimal gallery (i.e. a gallery of minimal length among the galleries of $\mathscr{A}$ from $C_{0}$ to $C_{n}$ ). From this perspective, the following lemma is a well known result.

Lemma 3.1. Let $F$ be a facet of $\mathscr{A}$, let $C$ and $D$ be two chambers having $F$ as facet, and let $f$ be a positive minimal path of $\Gamma(\mathscr{A})$ beginning at $C$ and ending in $D$. Then $f$ is a path of $\Gamma_{F}$.

Lemma 3.2. Let $\Phi^{o}$ be a simplex of $\operatorname{Del}^{o}(\mathscr{A})$. Then $\hat{\Gamma}_{\Phi^{o}}$ is a connected component of $\rho^{-1}\left(\Gamma_{F\left(\Phi^{o}\right)}\right)$.

Proof. Fix a vertex $v_{0}$ of $\hat{\Gamma}_{\Phi^{o}}$. Let $\Theta$ denote the connected component of $\rho^{-1}\left(\Gamma_{F\left(\Phi^{o}\right)}\right)$ with $v_{0} \in V(\Theta)$. Let us prove that $V(\Theta)=V\left(\hat{\Gamma}_{\Phi^{o}}\right)$.

Let $w \in V\left(\hat{\Gamma}_{\Phi^{o}}\right)$. Choose a point $\alpha^{o} \in \Phi^{o}$, and write $\alpha=\pi^{o}\left(\alpha^{0}\right)$. Since $\alpha^{o} \in$ $\Delta_{l-1}^{o}\left(v_{0}\right) \cap \Delta_{l-1}^{o}(w)$, by definition of $\operatorname{Del}^{o}(\mathscr{A})$, there exists a path $f=a_{1}^{\varepsilon_{1}} \ldots a_{n}^{\varepsilon_{n}}$ of $\hat{\Gamma}(\mathscr{A})$ beginning at $v_{0}$, ending in $w$, and such that $\alpha \in \Delta_{l-1}\left(\rho\left(v_{i}\right)\right)$ for every $i=0$, $1, \ldots, n$, where $v_{i}=\operatorname{end}\left(a_{1}^{\varepsilon_{1}} \ldots a_{i}^{\varepsilon_{i}}\right)$ for $i=0,1, \ldots, n$. We have $\alpha \in \pi^{\circ}\left(\Phi^{\circ}\right) \cap$ $\Delta_{l-1}\left(\rho\left(v_{i}\right)\right) \subseteq F\left(\Phi^{0}\right) \cap \bar{\rho}\left(v_{i}\right)$, where $\bar{\rho}\left(v_{i}\right)$ is the closure of $\rho\left(v_{i}\right)$ in $V$, thus $F\left(\Phi^{o}\right) \cap \bar{\rho}\left(v_{i}\right) \neq \emptyset$, and therefore $F\left(\Phi^{0}\right)$ is a facet of $\rho\left(v_{i}\right)$ for every $i=0,1, \ldots, n$. This implies that $\rho\left(v_{i}\right) \in V\left(\Gamma_{F\left(\Phi^{o}\right)}\right)$, thus $\rho(f)$ is a path of $\Gamma_{F\left(\Phi^{o}\right)}$, and therefore $f$ is a path of $\Theta$ (since origin $(f)=v_{0} \in V(\Theta)$ ). It follows that end $(f)=w \in$ $V(\Theta)$.

Now, let $w \in V(\Theta)$. Choose a path $f=a_{1}^{\varepsilon_{1}} \ldots a_{n}^{\varepsilon_{n}}$ of $\Theta$ beginning at $v_{0}$ and ending in $w$. Write $v_{i}=\operatorname{end}\left(a_{1}^{\varepsilon_{1}} \ldots a_{i}^{\varepsilon_{i}}\right)$ for $i=0,1, \ldots, n$. We have $\pi^{o}\left(\Phi^{o}\right) \subseteq$ $\Delta_{l-1}\left(\rho\left(v_{i}\right)\right) \cap \Delta_{l-1}\left(\rho\left(v_{i+1}\right)\right)$ for $i=0,1, \ldots, n-1$ (since $\rho(f)$ is a path of $\left.\Gamma_{F\left(\Phi^{o}\right)}\right)$, thus, by the definition of $\operatorname{Del}^{o}(\mathscr{A})$, we successively have $\Phi^{o} \subseteq \Delta_{l-1}^{o}\left(v_{\imath}\right)$ for $i=0,1, \ldots, n$. In particular, $\Phi^{o} \subseteq \Delta_{l-1}^{o}(w)$, namely, $w \in V\left(\hat{\Gamma}_{\Phi^{o}}\right)$. 


\section{Part 2.}

For a simplex $\phi$ of $\mathbf{S}^{l-1}$, we denote by $K(\phi)$ the cone over $\phi$;

$$
K(\phi)=\{\lambda x \mid \lambda>0 \text { and } x \in \phi\} .
$$

Note that $K(\phi) \subseteq F(\phi)$ for every simplex $\phi$ of $\mathbf{S}^{l-1}$, and $\{K(\phi) \mid \phi$ a simplex of $\left.\mathbf{S}^{l-1}\right\}$ is a partition of $V-\{0\}$.

Let $S$ be a simplicial complex, and let $\phi$ and $\phi$ be two simplexes of $S$. We set $\phi \geq \phi$ if $\bar{\phi} \supset \phi$, where $\bar{\phi}$ is the closure of $\phi$ in $S$. The relation " $\geq$ " is a partial order in the set of simplexes of $S$.

Recall that, for a chamber $C$ of $\mathscr{A}$ and for a facet $F$, we denote by $C_{F}$ the unique chamber of $\mathscr{A}_{|F|}$ containing $C$.

For a simplex $\phi$ of $\mathbf{S}^{l-1}$ and for a chamber $C$ of $\mathscr{A}$, we write

$$
R(\phi, C)=\bigcup_{\psi \geq \phi}\left(K(\phi)+i C_{F(\psi)}\right) .
$$

We have $R(\phi, C) \subseteq M(C)$.

Lemma 3.3. Let $\phi$ be a simplex of $\mathbf{S}^{l-1}$, and let $C$ be a chamber of $\mathscr{A}$. Then $R(\phi, C)$ is an open subset of $M(\mathscr{A})$.

Proof. Pick $z=(x+i y) \in R(\phi, C)$. Let $\phi$ be the simplex of $\mathbf{S}^{l-1}$ such that $x \in K(\phi)$. Then we have $y \in C_{F(\psi)}$. If $\phi^{\prime} \geq \phi$, then $F\left(\phi^{\prime}\right) \geq F(\phi)$, thus $C_{F\left(\phi^{\prime}\right)} \supseteq$ $C_{F(\psi)}$. Furthermore, the subset $\cup_{\psi^{\prime} \geq \psi} K\left(\phi^{\prime}\right)$ is an open cone. It follows that

$$
T(z)=\left(\underset{\phi^{\prime} \geq \psi}{\bigcup} K\left(\phi^{\prime}\right)\right)+i C_{F(\psi)}
$$

is an open neighbourhood of $z$, and $T(z) \subseteq R(\phi, C)$.

Recall that, for every chamber $C$ of $\mathscr{A}$,

$$
q^{-1}(M(C))=\underset{v \in \rho^{-1}(C)}{\bigcup} \hat{M}(v),
$$

this union is disjoint, and $q$ sends $\hat{M}(v)$ homeomorphically onto $M(v)=M(C)$ for every $v \in \rho^{-1}(C)$ (see Lemma 2.7). For a simplex $\phi$ of $\mathbf{S}^{l-1}$ and for a vertex $v$ of $\hat{\Gamma}(\mathscr{A})$, we denote by $\hat{R}(\phi, v)$ the lift of $R(\phi, \rho(v))$ into $M(v)$. By Lemma 3.3, $\hat{R}(\phi, v)$ is an open subset of $\hat{\Gamma}(\mathscr{A})$.

Now, let us define $U(\Phi)$, where $\Phi$ is a simplex of $\operatorname{Del}(\mathscr{A})$.

If $\Phi$ is a simplex of $\operatorname{Del}^{\circ}(\mathscr{A})$, then 


$$
U(\Phi)=\bigcup_{v} \hat{R}\left(\pi^{o}(\Phi), v\right)
$$

where the union is over all the vertices of $\hat{\Gamma}_{\Phi}$.

Assume that $\Phi=\omega\left(\mathbf{B}^{l}(v)\right)$, where $v$ is a vertex of $\hat{\Gamma}(\mathscr{A})$. Write $C=\rho(v)$. The set $U(\Phi)=U\left(\omega\left(\mathbf{B}^{l}(v)\right)\right)$ is the lift of $(V+i C) \subseteq M(C)$ into $\hat{M}(v)$.

Assume that $\Phi$ has the form $\Phi=\Phi^{o} \vee \omega\left(\mathbf{B}^{l}(v)\right)$, where $v$ is a vertex of $\hat{\Gamma}(\mathscr{A})$ and $\Phi^{o}$ is a simplex of $\operatorname{Del}^{o}(\mathscr{A})$ contained in $\mathbf{S}^{l-1}(v)$. Write $\phi=\pi^{o}\left(\Phi^{o}\right)$ and $C=\rho(v)$. Then $U(\Phi)$ is the lift of

$$
\left(\bigcup_{\psi \geq \psi} K(\phi)\right)+i C \subseteq M(C)
$$

into $\hat{M}(v)$.

\section{Part 3.}

Lemma 3.4. i) Let $\omega_{0}, \omega_{1}, \ldots, \omega_{r}$ be $(r+1)$ vertices of $\operatorname{Del}^{o}(\mathscr{A})$. If $\cap_{i=0}^{r} U\left(\omega_{i}\right) \neq \emptyset$, then $\omega_{0}, \omega_{1}, \ldots, \omega_{r}$ are the vertices of a simplex $\Phi^{o}$ of $\operatorname{Del}^{o}(\mathscr{A})$.

ii) Let $\omega_{0}, \omega_{1}, \ldots, \omega_{r}$ be the vertices of a simplex $\Phi^{o}$ of $\operatorname{Del}^{o}(\mathscr{A})$. Then $\cap_{i=0}^{r} U\left(\omega_{i}\right)=U\left(\Phi^{o}\right)$.

Proof. i) Let $\omega_{0}, \omega_{1}, \ldots, \omega_{r}$ be $(r+1)$ vertices of $\operatorname{Del}^{o}(\mathscr{A})$ such that $\cap_{i=0}^{r} U\left(\omega_{i}\right) \neq \varnothing$. Write $x_{i}=\pi^{o}\left(\omega_{i}\right)$ for $i=0,1, \ldots, r$. Pick $e \in \cap_{i=0}^{r} U\left(\omega_{i}\right)$. Write $z=(x+i y)=q(e)$. For every $i=0,1, \ldots, r$, we choose a vertex $v_{i}$ of $\hat{\Gamma}_{\omega_{i}}$ such that $e \in \hat{R}\left(x_{i}, v_{i}\right)$, and we write $A_{i}=\rho\left(v_{i}\right)$.

Let $\phi$ be the simplex of $\mathbf{S}^{l-1}$ such that $x \in K(\phi)$. By the definition of $R\left(x_{i}\right.$, $A_{i}$ ), we have $\phi \geq x_{i}$ for $i=0,1, \ldots, r$, thus $x_{0}, x_{1}, \ldots, x_{r}$ are vertices of $\phi$.

By the definition of $R\left(x_{i}, A_{i}\right)$, we have $y \in\left(A_{i}\right)_{F(\psi)}$ for every $i=0,1, \ldots, r$, thus $\bigcap_{i=0}^{r}\left(A_{i}\right)_{F(\psi)} \neq \varnothing$, therefore $\left(A_{0}\right)_{F(\psi)}=\left(A_{1}\right)_{F(\psi)}=\ldots=\left(A_{r}\right)_{F(\psi)}$. Let $C$ be the chamber of $\mathscr{A}$ having $F(\phi)$ as facet and such that $C_{F(\psi)}=\left(A_{0}\right)_{F(\phi)}=\ldots=$ $\left(A_{r}\right)_{F(\psi)}$.

Let $i \in\{0,1, \ldots, r\}$. The facet $F\left(x_{i}\right)$ of $\mathscr{A}$ is common to $A_{i}$ and $C$ (since $\left.F(\phi) \geq F\left(x_{i}\right)\right)$. We fix a positive minimal path $f_{i}$ of $\Gamma(\mathscr{A})$ beginning at $A_{i}$ and ending in $C$. By Lemma 3.1, $f_{i}$ is a path of $\Gamma_{F\left(x_{i}\right)}$. We denote by $\hat{f}_{i}$ the lift of $f_{\imath}$ into $\hat{\Gamma}(\mathscr{A})$ beginning at $v_{i}$. By Lemma 3.2, $\hat{f}_{i}$ is a path of $\hat{\Gamma}_{\omega_{i}}$.

Write $w=\operatorname{end}\left(\hat{f}_{0}\right)$. First, let us prove that $w=\operatorname{end}\left(\hat{f}_{i}\right)$ for every $i=1, \ldots, r$. By Lemma 2.6, we have $z \in R\left(x_{0}, v_{0}\right) \cap R\left(x_{i}, v_{i}\right) \cap\left(Z\left(v_{0}, v_{i}\right)+i V\right)$, therefore $x \in Z\left(v_{0}, v_{i}\right)$. Furthermore, $x \in F(\phi)$ and $Z\left(v_{0}, v_{i}\right)$ is a union of facets of $\mathscr{A}$, thus $F(\phi) \subseteq Z\left(v_{0}, v_{i}\right)$. Finally $F(\phi) \subseteq \bar{C}$ and $Z\left(v_{0}, v_{1}\right)$ is an open subset of $V$, 
therefore $C \subseteq Z\left(v_{0}, v_{i}\right)$. Thus, by the construction of $Z\left(v_{0}, v_{i}\right)$, there exists a vertex $u_{i} \in \sum\left(v_{0}\right) \cap \sum\left(v_{i}\right)$ such that $\rho\left(u_{i}\right)=C$. This can happen only if $u_{i}=$ $\operatorname{end}\left(\hat{f}_{0}\right)=\operatorname{end}\left(\hat{f}_{1}\right)$.

Now, consider the simplex $\Psi^{0}$ of $\operatorname{Del}^{0}(\mathscr{A})$ such that $\Psi^{0} \subseteq \Delta_{l-1}^{0}(w)$ and $\pi^{0}\left(\Psi^{0}\right)=\phi$. Let us show that $\omega_{\imath}$ is a vertex of $\Psi^{0}$ for every $i=0,1, \ldots, r$. Recall that $\hat{f}_{i}$ is a path of $\hat{\Gamma}_{\omega_{i}}$, thus end $\left(\hat{f}_{i}\right)=\omega \in V\left(\hat{\Gamma}_{\omega_{i}}\right)$, therefore $\omega_{i} \in$ $\Delta_{l=1}^{o}(w)$. It follows that $\omega_{l}$ is the unique vertex of $\Psi^{0} \subseteq \Delta_{l-1}^{o}(w)$ such that $\pi^{o}\left(\omega_{i}\right)=x_{i}$

ii) Let $\omega_{0}, \omega_{1}, \ldots, \omega_{r}$ be the vertices of a simplex $\Phi^{o}$ of $\operatorname{Del}^{o}(\mathscr{A})$. Write $x_{i}=$ $\pi^{o}\left(\omega_{i}\right)$ for $i=0,1, \ldots, r$, and $\phi=\pi^{o}\left(\Phi^{o}\right)$.

Let $e \in U_{i=0}^{r} U\left(\omega_{i}\right)$. Write $z=(x+i y)=q(e)$. For every $i=0,1, \ldots, r$, we choose a vertex $v_{i}$ of $\hat{\Gamma}_{\omega_{i}}$ such that $e \in \hat{R}\left(x_{\imath}, v_{\imath}\right)$, and we write $A_{\imath}=\rho\left(v_{i}\right)$. Let $w$ be the vertex of $\hat{\Gamma}(\mathscr{A})$ defined in the proof of i). Let us prove that $w \in V\left(\hat{\Gamma}_{\Phi^{o}}\right)$ and $e \in \hat{R}(\phi, w)$. This shows that $e \in U\left(\Phi^{\circ}\right)$.

Consider the simplex $\Psi^{o}$ defined in the proof of i), and write $\phi=\pi^{o}\left(\Psi^{o}\right)$. The simplex $\psi$ is the (unique) simplex of $\mathbf{S}^{l-1}$ such that $x \in K(\phi)$. Since $\omega_{0}, \omega_{1}, \ldots, \omega_{r}$ are vertices of $\Psi^{o}$, we have $\Psi^{o} \geq \Phi^{o}$, thus $V\left(\hat{\Gamma}_{\Psi^{o}}\right) \subseteq V\left(\hat{\Gamma}_{\Phi^{o}}\right)$, there fore $w \in V\left(\hat{\Gamma}_{\Phi^{o}}\right)$ (since $w \in V\left(\hat{\Gamma}_{\psi^{o}}\right)$ ).

In order to prove that $e \in \hat{R}(\phi, w)$, by Lemma 2.6, it suffices to show that

$$
z \in R\left(x_{0}, A_{0}\right) \cap R(\phi, C) \cap\left(Z\left(v_{0}, w\right)+i V\right),
$$

where $A_{0}=\rho\left(v_{0}\right)$ and $C=\rho(w)$. By the starting hypothesis, we have $z \in R\left(x_{0}, A_{0}\right)$. The inequality $\phi \geq \phi$ and the inclusions $x \in K(\phi)$ and $y \in C_{F(\psi)}$ $=\left(A_{0}\right)_{F(\psi)}$ imply $z \in R(\phi, C)$. Now, $C \subseteq Z\left(v_{0}, w\right)$ (since $w \in \sum\left(v_{0}\right) \cap \sum(w)$ ) and $F(\phi) \subseteq \bar{C}$, thus $F(\phi) \subseteq \bar{Z}\left(v_{0}, w\right)$. Since $\left(A_{0}\right)_{F(\phi)}=C_{F(\psi)}$, no hyperplane of $\mathscr{A}$ which separates $A_{0}$ and $C$ contains $F(\phi)$, thus, by Lemma 2.5, $x \in F(\phi) \subseteq$ $Z\left(v_{0}, w\right)$. It follows that $z=(x+i y) \in\left(Z\left(v_{0}, w\right)+i V\right)$.

Now, let $e \in U\left(\Phi^{o}\right)$. We choose a vertex $v$ of $\hat{\Gamma}_{\Phi^{o}}$ such that $e \in \hat{R}(\phi, v)$. Then we have $v \in V\left(\hat{\Gamma}_{\omega_{i}}\right)$ and $\hat{R}(\phi, v) \subseteq \hat{R}\left(x_{i}, v\right)$ for every $i=0,1, \ldots, r$, thus $e \in \cap_{\imath=0}^{r} U\left(\omega_{\imath}\right)$.

Lemma 3.5. i) Let $v$ and $w$ be two vertices of $\hat{\Gamma}(\mathscr{A})$. If $v \neq w$, then $U\left(\omega\left(\mathbf{B}^{l}(v)\right)\right) \cap U\left(\omega\left(\mathbf{B}^{l}(w)\right)\right)=\emptyset$.

ii ) Let $\Phi^{o}$ be a simplex of $\operatorname{Del}^{o}(\mathscr{A})$, and let $v$ be a vertex of $\hat{\Gamma}(\mathscr{A})$. If $U\left(\Phi^{o}\right) \cap$ $U\left(\omega\left(\mathbf{B}^{l}(v)\right)\right) \neq \varnothing$, then $\Phi^{o} \subseteq \mathbf{S}^{l-1}(v)$.

iii) Let $v$ be a vertex of $\hat{\Gamma}(\mathscr{A})$, and let $\Phi^{o}$ be a simplex of $\operatorname{Del}^{\circ}(\mathscr{A})$ such that $\Phi^{o} \subseteq \mathbf{S}^{l-1}(v)$. Write $\Phi=\Phi^{o} \vee \omega\left(\mathbf{B}^{l}(v)\right)$. Then $U\left(\Phi^{o}\right) \cap U\left(\omega\left(\mathbf{B}^{l}(v)\right)\right)=U(\Phi)$. 
Proof. i ) Let $v$ and $w$ be two vertices of $\hat{\Gamma}(\mathscr{A})$. Assume $U\left(\omega\left(\mathbf{B}^{l}(v)\right)\right) \cap$ $U\left(\omega\left(\mathbf{B}^{l}(w)\right)\right) \neq \emptyset$, and let us prove that $v=w$.

We have

$$
\begin{aligned}
& q\left(U\left(\omega\left(\mathbf{B}^{l}(v)\right)\right)\right) \cap q\left(U\left(\omega\left(\mathbf{B}^{l}(w)\right)\right)\right)=(V+i \rho(v)) \cap(V+i \rho(w)) \neq \emptyset \\
\Rightarrow & \rho(v) \cap \rho(w) \neq \emptyset \\
\Rightarrow & \rho(v)=\rho(w) .
\end{aligned}
$$

Write $C=\rho(v)=\rho(w)$. We know that

$$
q^{-1}(M(C))=\bigcup_{u \in \rho^{-1}(C)} \hat{M}(u),
$$

this union is disjoint, $U\left(\omega\left(\mathbf{B}^{l}(v)\right)\right) \subseteq \hat{M}(v)$, and $U\left(\omega\left(\mathbf{B}^{l}(w)\right)\right) \subseteq \hat{M}(w)$. Thus $v=w$.

ii) Let $v$ be a vertex of $\hat{\Gamma}(\mathscr{A})$, and let $\Phi^{\circ}$ be a simplex of $\operatorname{Del}^{\circ}(\mathscr{A})$. Assume $U\left(\Phi^{o}\right) \cap U\left(\omega\left(\mathbf{B}^{l}(v)\right)\right) \neq \emptyset$. Write $\phi=\pi^{o}\left(\Phi^{o}\right)$. Pick an $e \in U\left(\Phi^{o}\right) \cap$ $U\left(\omega\left(\mathbf{B}^{l}(v)\right)\right)$, and write $z=(x+i y)=q(e)$. We choose a vertex $w$ of $\hat{\Gamma}_{\Phi^{o}}$ such that $e \in \hat{R}(\phi, w)$. We write $A=\rho(v)$ and $B=\rho(w)$. Let $\phi$ be the simplex of $\mathbf{S}^{l-1}$ such that $x \in K(\phi)$.

We have $y \in A$ (since $z \in(V+i A)$ ) and $y \in B_{F(\phi)}$ (since $z \in R(\phi, B)$ ), thus $A_{F(\psi)} \cap B_{F(\psi)} \neq \emptyset$, therefore $A_{F(\psi)}=B_{F(\psi)}$. Let $C$ be the chamber of $\mathscr{A}$ having $F(\phi)$ as facet and such that $C_{F(\psi)}=A_{F(\phi)}=B_{F(\phi)}$. Let $f$ be a positive minimal path of $\Gamma(\mathscr{A})$ beginning at $A$ and ending in $C$, and let $g$ be a positive minimal path of $\Gamma(\mathscr{A})$ beginning at $B$ and ending in $C$. By the definition of $R(\phi, B)$, we have $\phi \geq \phi$ (since $(x+i y) \in R(\phi, B)$ and $x \in K(\phi)$ ), thus $F(\phi) \geq F(\phi)$, therefore $F(\phi)$ is a facet of $C$. On the other hand, we have $\Phi^{o} \subseteq \Delta_{l-1}^{o}(w)$, thus $F\left(\Phi^{o}\right)=$ $F(\phi)$ is a facet of $\rho(w)=B$. It follows that $B$ and $C$ are vertices of $\Gamma_{F(\phi)}$ and, consequently, by Lemma $3.1, g$ is a path of $\Gamma_{F(\phi)}$.

We denote by $\hat{f}$ the lift of $f$ into $\hat{\Gamma}(\mathscr{A})$ beginning at $v$, and by $\hat{g}$ the lift of $g$ into $\hat{\Gamma}(\mathscr{A})$ beginning at $w$. First, let us prove that end $(\hat{f})=\operatorname{end}(\hat{g})$. By Lemma 2.6 , we have

$$
z=(x+i y) \in(V+i A) \cap R(\phi, B) \cap(Z(v, w)+i V),
$$

thus $x \in Z(v, w)$. Furthermore, $x \in F(\phi)$ and $Z(v, w)$ is a union of facets of $\mathscr{A}$, thus $F(\phi) \subseteq Z(v, w)$. Finally, $F(\phi) \subseteq \bar{C}$ and $Z(v, w)$ is an open subset of $V$, therefore $C \subseteq Z(v, w)$. This implies, by the definition of $Z(v, w)$, that there exists a vertex $u \in \sum(v) \cap \sum(w)$ such that $\rho(u)=C$. This can happen only if $\operatorname{end}(\hat{f})=\operatorname{end}(\hat{g})=u$.

Now, let us prove that $\Phi^{o} \subseteq \Delta_{l-1}^{o}(u) \subseteq \mathbf{S}^{l-1}(v)$. The path $g$ is a path of 
$\Gamma_{F\left(\Phi^{0}\right)}=\Gamma_{F(\phi)}$, the vertex $w$ is a vertex of $\hat{\Gamma}_{\Phi^{o}}$, and $\hat{\Gamma}_{\Phi^{o}}$ is a connected component of $\rho^{-1}\left(\Gamma_{F\left(\Phi^{o}\right)}\right)$ (Lemma 3.2), thus $\hat{g}$ is a path of $\hat{\Gamma}_{\Phi^{0}}$, and, consequently, $u=\operatorname{end}(\hat{g}) \in$ $V\left(\hat{\Gamma}_{\Phi^{o}}\right)$. It follows, by the definition of $\hat{\Gamma}_{\Phi^{o}}$, that $\Phi^{o} \subseteq \Delta_{l-1}^{o}(u)$. On the other hand, $u \in \Sigma(v)$, therefore, by the definition of $\mathbf{S}^{l-1}(v)$, we have $\Delta_{l-1}^{o}(u) \subseteq \mathbf{S}^{l-1}(v)$.

iii) Let $v$ be a vertex of $\hat{\Gamma}(\mathscr{A})$, and let $\Phi^{o}$ be a simplex of $\operatorname{Del}^{o}(\mathscr{A})$ such that $\Phi^{o} \subseteq \mathbf{S}^{l-1}(v)$. We write $\Phi=\Phi^{o} \vee \omega\left(\mathbf{B}^{l}(v)\right)$ and $\phi=\pi^{o}\left(\Phi^{o}\right)$.

Let $e \in U\left(\Phi^{o}\right) \cap U\left(\omega\left(\mathbf{B}^{l}(v)\right)\right)$. Pick a vertex $w$ of $\hat{\Gamma}_{\Phi^{o}}$ such that $e \in \hat{R}(\phi, w)$. Write $A=\rho(v)$ and $B=\rho(w)$. We have

$$
\begin{aligned}
& e \in U\left(w\left(\mathbf{B}^{l}(v)\right)\right) \cap \hat{R}(\phi, w) \\
\Rightarrow & q(e) \in(V+i A) \cap R(\phi, B) \cap(Z(v, w)+i V) \quad(\text { Lemma 2.6) } \\
\Rightarrow \quad & q(e) \in\left(\left(\cap_{\psi \geq \phi} K(\phi)\right)+i A\right) \cap R(\phi, B) \cap(Z(v, w)+i V) \\
& \left.\quad \quad \text { indeed, if }(x+i y) \in R(\phi, B), \text { then } x \in \cap_{\varphi \geq \phi} K(\phi)\right) \\
\Rightarrow & e \in U(\Phi) \cap \hat{R}(\phi, B) \quad(\text { Lemma 2.6) } \\
\Rightarrow & e \in U(\Phi) .
\end{aligned}
$$

Now, let $e \in U(\Phi)$. Write $z=(x+i y)=q(e)$ and $A=\rho(v)$. Let $\phi$ be the simplex of $\mathbf{S}^{l-1}$ such that $x \in K(\phi)$, and let $B$ be the chamber of $\mathscr{A}$ having $F(\phi)$ as facet and such that $A_{F(\psi)}=B_{F(\phi)}$. Pick a positive minimal path $f$ of $\Gamma(\mathscr{A})$ beginning at $A$ and ending in $B$, and denote by $\hat{f}$ the lift of $f$ into $\hat{\Gamma}(\mathscr{A})$ beginning at $v$. Set $w=\operatorname{end}(\hat{f})$. Let us prove that $w \in V\left(\hat{\Gamma}_{\Phi^{o}}\right)$ and $e \in \hat{R}(\phi, w)$, This shows that $e \in U\left(\Phi^{\circ}\right)$, and, consequently, $e \in U\left(\Phi^{\circ}\right) \cap$ $U\left(\omega\left(\mathbf{B}^{l}(v)\right)\right.$ ) (we obviously have $e \in U(\Phi) \subseteq U\left(\omega\left(\mathbf{B}^{l}(v)\right)\right.$ ).

Since $\phi \geq \phi$ and $\phi \subseteq \Delta_{l-1}(B)$, we have $\phi \subseteq \Delta_{l-1}(B)$. Thus there exists a simplex $\phi^{\prime 0} \subseteq \Delta_{l-1}^{o}(w)$ such that $\pi^{o}\left({\phi^{\prime}}^{0}\right)=\phi$. Moreover, $\Delta_{l-1}^{o}(w) \subseteq \mathbf{S}^{l-1}(v)$ (since $w \in \Sigma(v))$ and the restriction of $\pi^{o}$ to $\mathbf{S}^{l-1}(v)$ is an isomorphism $\mathbf{S}^{l-1}(v) \rightarrow \mathbf{S}^{l-1}$, therefore $\phi^{\prime 0}=\Phi^{o}$. It follows that $w \in V\left(\hat{\Gamma}_{\Phi^{0}}\right)$.

In order to prove that $e \in \hat{R}(\phi, w)$, by Lemma 3.6, it suffices to show that

$$
z \in(V+i A) \cap R(\phi, B) \cap(Z(v, w)+i V) .
$$

By the starting hypothesis, we have $z \in(V+i A)$ and $z=(x+i y) \in(K(\phi)+$ $\left.i B_{F(\psi)}\right) \subseteq R(\phi, B)$. Now, $w \in \sum(v) \cap \sum(w)$, thus $C \in Z(v, w)$. Moreover, $F(\phi) \subseteq \bar{C}$, therefore $F(\phi) \subseteq \bar{Z}(v, w)$. Finally, since $A_{F(\phi)}=B_{F(\psi)}$, no hyperplane of $\mathscr{A}$ containing $F(\phi)$ separates $A$ and $B$, thus, by Lemma 2.5, $x \in F(\phi) \subseteq$ $Z(v, w)$, therefore $z \in(Z(v, w)+i V)$.

Lemma 3.6. The set $\mathcal{U}=\{U(\omega) \mid \omega$ a vertex of $\operatorname{Del}(\mathscr{A})\}$ is a covering of $\hat{M}(\mathscr{A})$. 
Proof. Let $e \in \hat{M}(\mathscr{A})$. Write $z=(x+i y)=q(e)$.

Case a : $x=0$.

Then there exists a chamber $C$ of $\mathscr{A}$ such that $y \in C$. We have $z=(x+i y)$ $\in(V+i C) \subseteq M(C)$. By Lemma 2.7,

$$
q^{-1}(M(C))=\underset{v \in \rho^{-1}(C)}{\bigcup} \hat{M}(v),
$$

and this union is disjoint, so there exists a unique vertex $v \in \rho^{-1}(C)$ such that $e \in q^{-1}(V+i C) \cap \hat{M}(v)=U\left(\omega\left(\mathbf{B}^{l}(v)\right)\right)$.

Case $\mathrm{b}: x \neq 0$.

Let $\phi$ be the simplex of $\mathbf{S}^{l-1}$ such that $x \in K(\phi)$. Let $C$ be the chamber of $\mathscr{A}$ having $F(\phi)$ as facet and such that $y \in C_{F(\phi)}$ (recall that $K(\phi) \subseteq F(\phi)$ ). We have $z=(x+i y) \in\left(K(\phi)+i C_{F(\phi)}\right) \subseteq R(\phi, C) \subseteq M(C)$. By Lemma 2.7,

$$
q^{-1}(M(C))=\underset{v \in \rho^{-1}(C)}{\bigcup} \hat{M}(v)
$$

and this union is disjoint, so there exists a vertex $v \in \rho^{-1}(C)$ such that $e \in$ $q^{-1}(R(\phi, C)) \cap \hat{M}(v)=\hat{R}(\phi, v)$. We have $\phi \subseteq \Delta_{l-1}(C)$, thus there exists a simplex $\Phi^{o} \subseteq \Delta_{l-1}^{o}(v)$ such that $\pi^{o}\left(\Phi^{o}\right)=\phi$. We have $e \in \hat{R}(\phi, v)$ and $v \in\left(\hat{\Gamma}_{\Phi^{o}}\right)$, therefore $e \in U\left(\Phi^{o}\right)$. By Lemma 3.4, $e \in U(\omega)$, where $\omega$ is any vertex of $\Phi^{o}$.

\section{Part 4.}

Lemma 3.7. i ) Let $v$ be a vertex of $\hat{\Gamma}(\mathscr{A})$. Then $U\left(\omega\left(\mathbf{B}^{\prime}(v)\right)\right)$ is contractible.

ii) Let $v$ be a vertex of $\hat{\Gamma}(\mathscr{A})$, and let $\Phi^{\circ}$ be a simplex of $\operatorname{Del}^{\circ}(\mathscr{A})$ contained in $\mathbf{S}^{l-1}(v)$. Write $\Phi=\Phi^{o} \vee \omega\left(\mathbf{B}^{l}(v)\right)$. Then $U(\Phi)$ is contractible.

Proof. i) Write $A=\rho(v)$. Then

$$
q\left(U\left(\omega\left(\mathbf{B}^{l}(v)\right)\right)=(V+i A)\right.
$$

is clearly contractible, thus the lift $U\left(\omega\left(\mathbf{B}^{l}(v)\right)\right)$ of $q\left(U\left(\omega\left(\mathbf{B}^{l}(v)\right)\right)\right)$ into $\hat{M}(v)$ is also contractible.

ii) Write $A=\rho(v)$ and $\phi=\pi^{o}\left(\Phi^{\circ}\right)$. Then

$$
q(U(\Phi))=\left(\bigcup_{\phi \geq \phi} K(\phi)\right)+i A
$$

is clearly contractible, thus the lift $U(\Phi)$ of $q(U(\Phi))$ into $\hat{M}(v)$ is also contracti- 
ble.

Lemma 3.8. Let $\Phi^{o}$ be a simplex of $\operatorname{Del}^{o}(\mathscr{A})$. Then $U\left(\Phi^{o}\right)$ is homotopically equivalent to $\hat{M}\left(\mathscr{A}_{X\left(\Phi^{\circ}\right)}\right)$.

Following Lemmas 3.9 and 3.10 are preliminary results to the proof of Lemma 3.8.

For a simplex $\phi$ of $\mathbf{S}^{l-1}$, we write

$$
W(\phi)=\underset{C}{\cup} R(\phi, C),
$$

where the union is over all the chambers $C$ of $\mathscr{A}$ having $F(\phi)$ as facet (i.e. over all the vertices of $\left.V\left(\Gamma_{F(\phi)}\right)\right)$. The set $W(\phi)$ is an open subset of $M(\mathscr{A})$. We denote by $\iota_{\phi}^{0}: W(\phi) \rightarrow M(\mathscr{A})$ the inclusion map of $W(\phi)$ into $M(\mathscr{A})$, by $\iota_{\phi}^{1}: M(\mathscr{A}) \rightarrow$ $M\left(\mathscr{A}_{X(\phi)}\right)$ the inclusion map of $M(\mathscr{A})$ into $M\left(\mathscr{A}_{X(\phi)}\right)$, and by $\iota_{\phi}=\iota_{\phi}^{1} \circ \iota_{\phi}^{0}: W(\phi) \rightarrow$ $M\left(\mathscr{A}_{X(\phi)}\right)$ the inclusion map of $W(\phi)$ into $M\left(\mathscr{A}_{X(\phi)}\right)$.

Lemma 3.9. Let $\phi$ be a simplex of $\mathbf{S}^{l-1}$. Then $\iota_{\phi}: W(\phi) \rightarrow M\left(\mathscr{A}_{X(\phi)}\right)$ is a homotopy equivalence.

Proof. We have to define a continuous family $\left(h_{t}\right)_{0 \leq t \leq 1}: M\left(\mathscr{A}_{X(\phi)}\right) \rightarrow$ $M\left(\mathscr{A}_{X(\phi)}\right)$ of maps such that:

a) $h_{0}(z)=z$ for all $z \in M\left(\mathscr{A}_{X(\phi)}\right)$,

b) $h_{1}(z) \in W(\phi)$ for all $z \in M\left(\mathscr{A}_{X(\phi)}\right)$,

c) $h_{t}(z) \in W(\phi)$ for all $z \in W(\phi)$ and all $t \in[0,1]$.

We set

$$
K=\bigcup_{\phi \geq \phi} K(\phi)
$$

and we fix a point $x_{0} \in \phi$. Since $K$ is an open cone of $V$ and $x_{0} \in K$, there exists a continuous map $\lambda: V \rightarrow\left[0,+\infty\right.$ [ such that $\left(x+\lambda(x) x_{0}\right) \in K$ for all $x \in V$.

For every $z=(x+i y) \in M\left(\mathscr{A}_{X(\phi)}\right)$ and for every $t \in[0,1]$, we set

$$
h_{t}(z)=\left(x+t \lambda(x) x_{0}\right)+i y .
$$

The family $\left(h_{t}\right)_{0 \leq t \leq 1}: M\left(\mathscr{A}_{X(\phi)}\right) \rightarrow V_{\mathbf{C}}$ is a continuous family of maps, and $h_{0}(z)=z$ for all $z \in M\left(\mathscr{A}_{X(\phi)}\right)$. It remains to prove: 
1) $h_{t}(z) \in M\left(\mathscr{A}_{X(\phi)}\right)$ for all $z \in M\left(\mathscr{A}_{X(\phi)}\right)$ and all $t \in[0,1]$,

2) $h_{1}(z) \in W(\phi)$ for all $z \in M\left(\mathscr{A}_{X(\phi)}\right)$,

3) $h_{t}(z) \in W(\phi)$ for all $z \in W(\phi)$ and all $t \in[0,1]$.

1) Let $z=(x+i y) \in M\left(\mathscr{A}_{X(\phi)}\right)$. Suppose that there exists a $t \in[0,1]$ such that $h_{t}(z) \notin M\left(\mathscr{A}_{X(\phi)}\right)$. Then there exists a hyperplane $H \in \mathscr{A}_{X(\phi)}$ such that $h_{t}(z) \in H_{\mathbf{C}}$ (i.e. $\left(x+t \lambda(x) x_{0}\right) \in H$ and $\left.y \in H\right)$. Since $x_{0} \in \phi \subseteq H$ and $H$ is a linear space, we have $x \in H$ and $y \in H$, thus $z \in H_{\mathbf{C}}$. This contradicts the fact $z \in M\left(\mathscr{A}_{X(\Phi)}\right)$.

2) Let $z=(x+i y) \in M\left(\mathscr{A}_{X(\phi)}\right)$. We have $\left(x+\lambda(x) x_{0}\right) \in K$, so there exists a simplex $\phi$ of $\mathbf{S}^{l-1}$ such that $\phi \geq \phi$ and $\left(x+\lambda(x) x_{0}\right) \in K(\phi)$.

Let $G$ be the facet of $\mathscr{A}_{X(\phi)}$ with $\phi \subseteq G$. Let us prove that $|G|=$ $|F(\phi)|$ (recall that $F(\phi)$ is a facet of $\mathscr{A}$ but not necessarily of $\mathscr{A}_{X(\phi)}$ ). If a hyperplane $H \in \mathscr{A}$ contains $F(\phi)$, then $H \supseteq X(\phi)$ (since $\phi \geq \phi$ ), thus $H$ is a hyperplane of $\mathscr{A}_{X(\phi)}$ containing $\phi$, therefore $H \supseteq G$. This shows that $|G| \subseteq|F(\phi)|$. If a hyperplane $H \in \mathscr{A}_{X(\Phi)}$ contains $G$, then $H \in \mathscr{A}$ and $H \supseteq F(\phi)$. This shows that $|F(\phi)| \subseteq|G|$.

Now, since $\left(x+\lambda(x) x_{0}\right)+i y \in M\left(\mathscr{A}_{X(\phi)}\right)$ and $\left(x+\lambda(x) x_{0}\right) \in G$, there exists a chamber $D$ of $\mathscr{A}_{|G|}=\mathscr{A}_{|F(\psi)|}$ such that $y \in D$. Let $C$ be the chamber of $\mathscr{A}$ having $F(\phi)$ as facet and such that $D=C_{F(\phi)}$. The inequality $\phi \geq \phi$ implies $F(\phi) \geq F(\phi)$, thus $C$ has also $F(\phi)$ as facet. It follows that $h_{1}(z) \in(K(\phi)+$ $\left.i C_{F(\psi)}\right) \subseteq R(\phi, C) \subseteq W(\phi)$

3) Let $z=(x+i y) \in W(\phi)$. There are a chamber $C \in V\left(\Gamma_{F(\phi)}\right)$ and a simplex $\phi \geq \phi$ of $\mathbf{S}^{l-1}$ such that $z \in\left(K(\phi)+i C_{F(\phi)}\right)$. Since $x_{0} \in \phi \subseteq$ $\bar{K}(\phi)$ (where $\bar{K}(\phi)$ is the closure of $K(\phi)$ in $V$ ) and $K(\phi)$ is a convex cone, we have $\left(x+t \lambda(x) x_{0}\right) \in K(\phi)$, thus $h_{t}(z)=\left(\left(x+t \lambda(x) x_{0}\right)+i y\right) \in(K(\phi)+$ $\left.i C_{F(\psi)}\right) \subseteq W(\phi)$ for every $t \in[0,1]$.

Let $\Phi^{o}$ be a simplex of $\operatorname{Del}^{0}(\mathscr{A})$. We denote by $q_{\Phi^{o}}: U\left(\Phi^{o}\right) \rightarrow M(\mathscr{A})$ the restriction of $q$ to $U\left(\Phi^{o}\right)$. Note that $q_{\Phi^{o}}$ can be viewed as a map $q_{\Phi^{o}}: U\left(\Phi^{o}\right) \rightarrow$ $W\left(\pi^{o}\left(\Phi^{o}\right)\right)$ onto $W\left(\pi^{o}\left(\Phi^{o}\right)\right)$.

Lemma 3.10. Let $\Phi^{o}$ be a simplex of $\operatorname{Del}^{o}(\mathscr{A})$. Then $q_{\Phi^{o}}: U\left(\Phi^{o}\right) \rightarrow$ $W\left(\pi^{o}\left(\Phi^{o}\right)\right)$ is a cover. 
Proof. Write $\phi=\pi^{o}\left(\Phi^{\circ}\right)$. In order to prove Lemma 3.10, it suffices to show, for every chamber $A$ of $\mathscr{A}$ having $F(\phi)$ as facet, that

$$
q_{\Phi^{o}}^{-1}(R(\phi, A))=\bigcup_{v} \hat{R}(\phi, v),
$$

where the union is over all the vertices $v$ of $\rho_{\Phi^{0}}^{-1}(A)$; indeed, this union is disjoint (Lemma 2.7), the sets $\hat{R}(\phi, v)$ are copies of $R(\phi, A)$, the map $q_{\phi^{\circ}}$ is surjective, and $\left\{R(\phi, A) \mid A \in V\left(\Gamma_{F(\phi)}\right)\right\}$ is a covering of $W(\phi)$ by open subsets.

Fix $A \in V\left(\Gamma_{F(\phi)}\right)$, and pick $e \in q_{\Phi^{0}}^{-1}(R(\phi, A))$. By the definition of $U\left(\Phi^{o}\right)$, there exists a vertex $w$ of $\hat{\Gamma}_{\Phi^{o}}$ such that $e \in R(\phi, w)$. On the other hand, by Lemma 2.7,

$$
q_{\Phi^{o}}^{-1}(R(\phi, A)) \subseteq q^{-1}(R(\phi, A))=\bigcup_{v \in \rho^{-1}(A)} \hat{R}(\phi, v),
$$

thus there exists a vertex $v \in \rho^{-1}(A)$ such that $e \in \hat{R}(\phi, v)$. Write $z=(x+i y)$ $=q(e)$ and $B=\rho(w)$. Let $\phi$ be the simplex of $\mathbf{S}^{l-1}$ such that $x \in K(\psi)$. Since $z \in R(\phi, A) \cap R(\phi, B)$, we have $y \in A_{F(\psi)} \cap B_{F(\psi)}$, thus $A_{F(\psi)}=B_{F(\psi)}$. Let $C$ be the chamber of $\mathscr{A}$ having $F(\phi)$ as facet and such that $C_{F(\psi)}=A_{F(\psi)}=B_{F(\phi)}$.

Let $f$ be a positive minimal path of $\Gamma(\mathscr{A})$ beginning at $A$ and ending in $C$, and let $g$ be a positive minimal path of $\Gamma(\mathscr{A})$ beginning at $B$ and ending in $C$. The facet $F(\phi)$ is common to $A$ (since $A \in V\left(\Gamma_{F(\phi)}\right)$, to $B$ (since $w \in V\left(\hat{\Gamma}_{\Phi^{o}}\right)$ ), and to $C$ (since $F(\phi) \geq F(\phi)$ ), so, by Lemma 3.1, the paths $f$ and $g$ are paths of $\Gamma_{F(\phi)}$.

Let $\hat{f}$ denote the lift of $f$ into $\hat{\Gamma}(\mathscr{A})$ beginning at $v$, and let $\hat{g}$ denote the lift of $g$ into $\hat{\Gamma}(\mathscr{A})$ beginning at $w$. Let us prove that end $(\hat{f})=$ end $(\hat{g})$. This shows that $v \in \rho_{\Phi^{0}}^{-1}(A)$, thus ends the proof of Lemma 3.10; indeed, $g f^{-1}$ is a path of $\Gamma_{F(\phi)}$, the oriented graph $\hat{\Gamma}_{\phi^{o}}$ is a connected component of $\rho^{-1}\left(\Gamma_{F(\phi)}\right)$ (Lemma 3.2), and $w$ $\in V\left(\hat{\Gamma}_{\Phi^{o}}\right)$, thus $\hat{g} \hat{f}^{-1}$ is a path of $\hat{\Gamma}_{\Phi^{o}}$, and, consequently, $v=\operatorname{end}\left(\hat{g} \hat{f}^{-1}\right) \in V\left(\hat{\Gamma}_{\Phi^{o}}\right)$.

By Lemma 2.6,

$$
z \in R(\phi, A) \cap R(\phi, B) \cap(Z(v, w)+i V),
$$

thus $x \in Z(v, w)$. Moreover, $Z(v, w)$ is a union of facets of $\mathscr{A}$ and $x \in F(\phi)$, therefore $F(\phi) \subseteq Z(v, w)$. Finally $Z(v, w)$, is an open subset of $V$ and $F(\phi) \subseteq \bar{C}$, thus $C \subseteq Z(v, w)$. By the definition of $Z(v, w)$, there exists a vertex $u \in \sum(v) \cap \sum(w)$ such that $\rho(u)=C$. This can happen only if $u=\operatorname{end}(\hat{f})=$ end $(\hat{g})$.

Proof of Lemma 3.8. Let $\Phi^{\circ}$ be a simplex of $\operatorname{Del}^{\circ}(\mathscr{A})$. Write $\phi=\pi^{\circ}\left(\Phi^{\circ}\right)$ and $X=X\left(\Phi^{\circ}\right)$. We denote by $q_{X}: \hat{M}\left(\mathscr{A}_{X}\right) \rightarrow M\left(\mathscr{A}_{X}\right)$ the universal cover of $M\left(\mathscr{A}_{X}\right)$. Since $q$ is the universal cover of $M(\mathscr{A})$ and $q_{X}$ is a cover, there exists a map 
$\hat{\iota}_{\Phi^{0}}: \hat{M}(\mathscr{A}) \rightarrow \hat{M}\left(\mathscr{A}_{X}\right)$ such that the following diagram commutes.

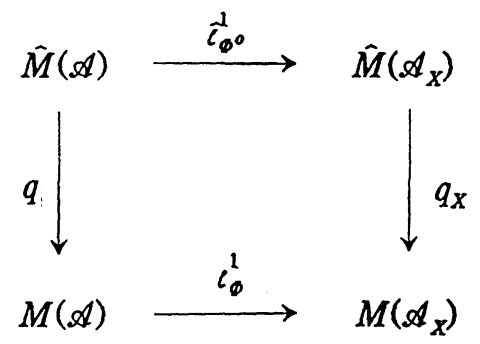

We denote by $\hat{\imath}_{\Phi^{o}}^{0}: U\left(\Phi^{o}\right) \rightarrow \hat{M}(\mathscr{A})$ the inclusion map of $U\left(\Phi^{o}\right)$ into $\hat{M}(\mathscr{A})$. Then the following diagram commutes.

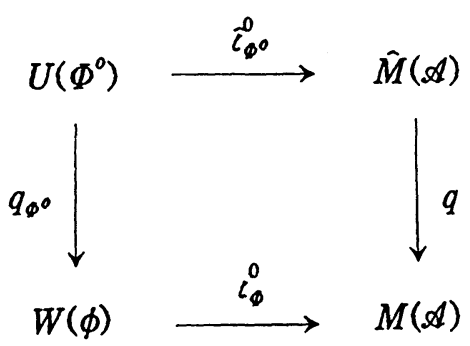

We write $\hat{\iota}_{\Phi^{o}}=\hat{\iota_{\Phi^{o}}} \circ \hat{\iota_{\Phi^{o}}}$. By the above considerations, the following diagram commutes.

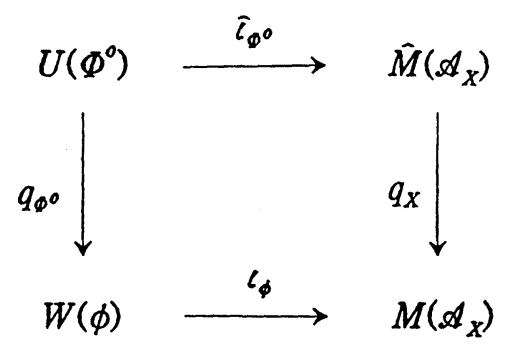

The map $\iota_{\phi}$ is a homotopy equivalence (Lemma 3.9), $q_{\Phi^{o}}$ is a cover (Lemma 3.10), and $q_{X}$ is the universal cover of $M\left(\mathscr{A}_{X}\right)$, thus $q_{\Phi^{\circ}}$ is the universal cover of $W(\phi)$ and $\hat{\iota}_{\Phi^{\circ}}$ is a homotopy equivalence.

Proposition 3.11. Let $\mathscr{A}$ be a real and essential arrangement of hyperplanes. Assume $\mathscr{A}_{X}$ to be a $K(\pi, 1)$ arrangement for every $X \in \mathscr{L}(\mathscr{A})$ different from $\{0\}$. 
Then $\operatorname{Del}(\mathscr{A})$ has the same homotopy type as the universal cover $\hat{M}(\mathscr{A})$ of $M(\mathscr{A})$.

Proof. Lemmas 3.4, 3.5 and 3.6 show that $\mathcal{U}=\{U(\omega) \mid \omega$ a vertex of $\operatorname{Del}(\mathscr{A})\}$ is a covering of $\hat{M}(\mathscr{A})$ having $\operatorname{Del}(\mathscr{A})$ as nerve. Lemmas 3.7 and 3.8 and the hypothesis " $\mathscr{A}_{X}$ is a $K(\pi, 1)$ arrangement for every $X \in \mathscr{L}(\mathscr{A})$ different from $\{0\}$ " show that every nonempty intersection of elements of $\mathcal{U}$ is contractible. It follows, by [We], that $\operatorname{Del}(\mathscr{A})$ is homotopically equivalent to $\hat{M}(\mathscr{A})$.

\section{Part 5.}

Proposition 3.12. Let $\mathscr{A}$ be a real and essential arrangement of hyperplanes. Assume that there exists an $X \in \mathscr{L}(\mathscr{A})$ different from $\{0\}$ such that $\mathscr{A}_{X}$ is not a $K(\pi, 1)$ arrangement. Then $\operatorname{Del}(\mathscr{A})$ is not homotopically equivalent to the universal cover $\hat{M}(\mathscr{A})$ of $M(\mathscr{A})$.

Proof. We are going to construct a space $\hat{M}_{\infty}$ by attaching cells to $\hat{M}(\mathscr{A})$, and a covering $\mathscr{U}_{\infty}=\left\{U_{\infty}(\omega) \mid \omega\right.$ a vertex of $\left.\operatorname{Del}(\mathscr{A})\right\}$ of $\hat{M}_{\infty}$ by open subsets, having $\operatorname{Del}(\mathscr{A})$ as nerve, and such that every nonempty intersection of elements of $U_{\infty}$ is contractible. By [We], the space $\hat{M}_{\infty}$ will be homotopically equivalent to $\operatorname{Del}(\mathscr{A})$. Afterwards, we will prove that there exists an integer $n_{0}>0$ such that the inclusion map $\hat{M}(\mathscr{A}) \rightarrow \hat{M}_{\infty}$ determines a surjective morphism $\pi_{n_{0}}(\hat{M}(\mathscr{A})) \rightarrow$ $\pi_{n_{0}}\left(\hat{M}_{\infty}\right)$ which is not injective. This shows that $\pi_{n_{0}}(\operatorname{Del}(\mathscr{A}))=\pi_{n_{0}}\left(\hat{M}_{\infty}\right) \neq$ $\pi_{n_{0}}(\hat{M}(\mathscr{A}))$.

Choose an $X \in \mathscr{L}(\mathscr{A})$ different from $\{0\}$ such that $\mathscr{A}_{X}$ is not a $K(\pi, 1)$ arrangement. Pick a simplex $\Phi^{o}$ of $\operatorname{Del}^{o}(\mathscr{A})$ such that $X\left(\Phi^{0}\right)=X$. By Lemma 3.8, $U\left(\Phi^{\circ}\right)$ has the same homotopy type as $\hat{M}\left(\mathscr{A}_{X}\right)$, so is not contractible.

It follows that there exists an integer $n_{0}>0$ such that:

i ) $\pi_{n}\left(U\left(\Phi^{o}\right)\right)=\{0\}$ for every simplex $\Phi^{o}$ of $\operatorname{Del}^{o}(\mathscr{A})$ and every $n \in\{0,1, \ldots$, $\left.n_{0}-1\right\}$

ii) there exists a simplex $\Phi^{o}$ of $\operatorname{Del}^{o}(\mathscr{A})$ such that $\pi_{n_{0}}\left(U\left(\Phi^{o}\right)\right) \neq\{0\}$. Recall that, if $\Phi$ is a simplex of $\operatorname{Del}(\mathscr{A})$ not contained in $\operatorname{Del}^{\circ}(\mathscr{A})$, then $U(\Phi)$ is contractible (Lemma 3.7 ).

We set $\hat{M}_{n_{0}-1}=\hat{M}(\mathscr{A})$, and $U_{n_{0}-1}(\Phi)=U(\Phi)$ for every simplex $\Phi$ of $\operatorname{Del}(\mathscr{A})$. 
First, we are going to define, by induction on $k \geq n_{0}$,

a) a space $\hat{M}_{k}$,

b) an open subspace $U_{k}(\Phi)$ of $\hat{M}_{k}$ for every simplex $\Phi$ of $\operatorname{Del}(\mathscr{A})$, such that:

1) $\hat{M}_{k-1} \subseteq \hat{M}_{k}$,

2) $U_{k-1}(\Phi)=U_{k}(\Phi) \cap \hat{M}_{k-1}$ for every simplex $\Phi$ of $\operatorname{Del}(\mathscr{A})$,

3) the inclusion map $\hat{M}_{k-1} \rightarrow \hat{M}_{k}$ induces an isomorphism of groups $\pi_{n}\left(\hat{M}_{k-1}\right) \rightarrow$ $\pi_{n}\left(\hat{M}_{k}\right)$ for every $n \in\{0,1, \ldots, k-1\}$, and induces a surjective morphism $\pi_{k}\left(\hat{M}_{k-1}\right) \rightarrow \pi_{k}\left(\hat{M}_{k}\right)$,

4) $\pi_{n}\left(U_{k}(\Phi)\right)=\{0\}$ for every simplex $\Phi$ of $\operatorname{Del}(\mathscr{A})$ and every $n \in\{0,1, \ldots, k\}$, 5) let $\omega_{0}, \omega_{1}, \ldots, \omega_{r}$ be $(r+1)$ vertices of $\operatorname{Del}(\mathscr{A})$, if $\cap_{j=0}^{r} U_{k}\left(\omega_{j}\right) \neq \emptyset$, then $\omega_{0}, \omega_{1}, \ldots, \omega_{r}$ are the vertices. of a simplex $\Phi$ of $\operatorname{Del}(\mathscr{A})$,

6) let $\omega_{0}, \omega_{1}, \ldots, \omega_{r}$ be the vertices of a simplex $\Phi$ of $\operatorname{Del}(\mathscr{A})$ then $\cap_{j=0}^{r} U_{k}\left(\omega_{j}\right)=$ $U_{k}(\Phi)$

7) $\left\{U_{k}(\Phi) \mid \omega\right.$ a vertex of $\left.\operatorname{Del}(\mathscr{A})\right\}$ is a covering of $\hat{M}_{k}$.

Assume $\hat{M}_{k-1}$ to be defined. Let $\Phi$ be a simplex of $\operatorname{Del}(\mathscr{A})$ such that $\pi_{k}\left(U_{k-1}(\Phi)\right) \neq\{0\}$. We fix a base point $e_{\Phi} \in U_{k-1}(\Phi)$. We choose a generator system $\left\{\gamma_{i}\right\}_{i \in I_{\Phi}}$ of $\pi_{r}\left(U_{k-1}(\Phi), e_{\Phi}\right)$, and, for every $i \in I_{\Phi}$, we fix a representative $\operatorname{map} f_{i}: \mathbf{S}^{k} \rightarrow U_{k-1}(\Phi)$ for $\gamma_{i}$. We write $I_{\Phi}=\emptyset$ if $\pi_{k}\left(U_{k-1}(\Phi)\right)=\{0\}$. We set

$$
I=\bigcup_{\Phi} I_{\Phi},
$$

where the union is over all the simplexes $\Phi$ of $\operatorname{Del}(\mathscr{A})$. The space $\hat{M}_{k}$ is obtained by attaching a $(k+1)$-cell $E_{i}$ to $\hat{M}_{k-1}$ by means of the map $f_{i}: \mathbf{S}^{k} \rightarrow \hat{M}_{k-1}$ defined on the boundary of $E_{i}$ for every $i \in I$. In other words, for every $i \in I$, we fix a copy $\mathbf{B}_{i}^{k+1}=\left\{x \in \mathbf{R}^{k+1} \mid\|x\| \leq 1\right\}$ of $\mathbf{B}^{k+1}$. Then

$$
\hat{M}_{k}=\left\{\hat{M}_{k-1} \amalg\left(\underset{i \in I}{\amalg} \mathbf{B}_{i}^{k+1}\right)\right\} / \sim,
$$

where $\sim$ is the equivalence relation on $\hat{M}_{k-1} \amalg\left(\amalg_{i \in I} \mathbf{B}_{i}^{k+1}\right)$ defined by $x \sim$ $f_{i}(x)$ for every $i \in I$ and for every $x \in \partial \mathbf{B}_{i}^{k+1}=\mathbf{S}^{k}$. We denote by $g_{\imath}: \mathbf{B}_{i}^{k+1} \rightarrow \hat{M}_{k}$ the natural map, and by $E_{i}$ the image of $g_{i}$ (where $i \in I$ ). We have $\left.g_{i}\right|_{\partial \mathbf{B}_{i}^{k+1}}=f_{i}$.

Let $\Phi$ be a simplex of $\operatorname{Del}(\mathscr{A})$. The set $U_{k}(\Phi)$ is defined by:

a) $U_{k}(\Phi) \cap \hat{M}_{k-1}=U_{k-1}(\Phi)$,

b) let $i \in I$, if $\partial E_{i} \subseteq U_{k-1}(\Phi)$, then $E_{i} \subseteq U_{k}(\Phi)$,

c) let $i \in I$, if $\partial E_{i} \nsubseteq U_{k-1}(\Phi)$, then

$$
U_{k}(\Phi) \cap E_{i}=g_{i}\left(\left\{\lambda x \mid 0<\lambda \leq 1 \text { and } x \in f_{i}^{-1}\left(U_{k-1}(\Phi)\right)\right\}\right) .
$$


Let $i \in I$, and let $\Phi$ be a simplex of $\operatorname{Del}(\mathscr{A})$. Then $g_{i}(0) \in U_{k}(\Phi)$ if and only if $\partial E_{i} \subseteq U_{k-1}(\Phi)$, and $g_{i}(\lambda x) \in U_{k}(\Phi)$ if and only if $g_{i}(x)=f_{i}(x) \in U_{k-1}(\Phi)$, where $\lambda \in[0,1]$ and $x \in \mathbf{S}^{l-1}$.

Now, let us prove Properties 1) to 7).

1) and 2) are obvious.

3) The space $\hat{M}_{k}$ is obtained by attaching $(k+1)$-cells to $\hat{M}_{k-1}$, so $\pi_{n}\left(\hat{M}_{k}\right.$, $\left.\hat{M}_{k-1}\right)=\{0\}$ for every $n \in\{0,1, \ldots, k\}$, thus the inclusion map $\hat{M}_{k-1} \rightarrow \hat{M}_{k}$ induces a group isomorphism $\pi_{n}\left(\hat{M}_{k-1}\right) \rightarrow \pi_{n}\left(\hat{M}_{k}\right)$ for every $n \in\{0,1, \ldots, k-1\}$, and induces a surjective morphism $\pi_{k}\left(\hat{M}_{k-1}\right) \rightarrow \pi_{k}\left(\hat{M}_{k}\right)$.

4) Let $\Phi$ be a simplex of $\operatorname{Del}(\mathscr{A})$. We denote by $U_{k}^{\prime}(\Phi)$ the subset of $\hat{M}_{k}$ defined by:

a) $U_{k}^{\prime}(\Phi) \cap \hat{M}_{k-1}=U_{k-1}(\Phi)$

b) let $i \in I$, if $\partial E_{i} \subseteq U_{k-1}(\Phi)$, then $E_{i} \subseteq U_{k}(\Phi)$,

c) let $i \in I$, if $\partial E_{i} \nsubseteq U_{k-1}(\Phi)$, then $\dot{E}_{i} \cap U_{k}^{\prime}(\Phi)=\emptyset$, where $\dot{E}_{i}$ is the interior of $E_{i}$.

The set $U_{k}^{\prime}(\Phi)$ is a strong deformation retract of $U_{k}(\Phi)$ and is obtained by attaching $(k+1)$-cells to $U_{k-1}(\Phi)$. If follows that the inclusion map $U_{k-1}(\Phi) \rightarrow$ $U_{k}(\Phi)$ induces a group isomorphism $\pi_{n}\left(U_{k-1}(\Phi)\right) \rightarrow \pi_{n}\left(U_{k}(\Phi)\right)$ for every $n \in\{0,1, \ldots, k-1\}$, and induces a surjective morphism $\xi_{k}^{\Phi}: \pi_{k}\left(U_{k-1}(\Phi)\right) \rightarrow$ $\pi_{k}\left(U_{k}(\Phi)\right)$. A first consequence is, by the inductive hypothesis, that $\pi_{n}\left(U_{k}(\Phi)\right)=$ $\pi_{n}\left(U_{k-1}(\Phi)\right)=\{0\}$ for every $n \in\{0,1, \ldots, k-1\}$. On the other hand, by the construction of $\hat{M}_{k}$, every generator $\gamma_{i}$ of $\pi_{k}\left(U_{k-1}(\Phi), e_{\Phi}\right)$ is sent by $\xi_{k}^{\Phi}$ onto 0 , thus the image of $\xi_{k}^{\Phi}$ is $\{0\}=\pi_{k}\left(U_{k}(\Phi)\right)$.

5) Let $\omega_{0}, \omega_{1}, \ldots, \omega_{r}$ be $(r+1)$ vertices of $\operatorname{Del}(\mathscr{A})$ such that $\cap_{j=0}^{r} U_{k}\left(\omega_{j}\right) \neq \emptyset$. Pick an $e \in \cap_{j=0}^{r} U_{k}\left(\omega_{j}\right)$.

Case a: $e \in \hat{M}_{k-1}$. Then $e \in \cap_{j=0}^{r} U_{k-1}\left(\omega_{j}\right)$, thus, by the inductive hypothesis, $\omega_{0}, \omega_{1}, \ldots, \omega_{r}$ are the vertices of a simplex $\Phi$ of $\operatorname{Del}(\mathscr{A})$.

Case b: There exists an $i \in I$ such that $e \in E_{i}$ and $e=g_{i}^{-1}(0)$. Then, by the construction of $U_{k}\left(\omega_{j}\right)$, we have $\partial E_{i} \in U_{k-1}\left(\omega_{j}\right)$ for every $j=0,1, \ldots, r$, therefore $\bigcap_{j=0}^{r} U_{k-1}\left(\omega_{j}\right) \neq \emptyset$. It follows, by the inductive hypothesis, that $\omega_{0}, \omega_{1}, \ldots, \omega_{r}$ are the vertices of a simplex $\Phi$ of $\operatorname{Del}(\mathscr{A})$.

Case c: There exists an $i \in I$ such that $e \in E_{i}$ and $e \neq g_{i}^{-1}(0)$. There are an $x \in \mathbf{S}^{k}$ and a $\left.\left.\lambda \in\right] 0,1\right]$ such that $e=g_{i}(\lambda x)$. By the construction of $U_{k}\left(\omega_{j}\right)$, we have $g_{i}(x)=f_{i}(x) \in U_{k-1}\left(\omega_{j}\right)$ for every $j=0,1, \ldots, r$, therefore 
$\bigcap_{j=0}^{r} U_{k-1}\left(\omega_{j}\right) \neq \varnothing$. It follows, by the inductive hypothesis, that $\omega_{0}, \omega_{1}, \ldots, \omega_{r}$ are the vertices of a simplex $\Phi$ of $\operatorname{Del}(\mathscr{A})$.

6) Let $\omega_{0}, \omega_{1}, \ldots, \omega_{r}$ be the vertices of a simplex $\Phi$ of $\operatorname{Del}(\mathscr{A})$.

a) $\left(\cap_{j=0}^{r} U_{k}\left(\omega_{j}\right)\right) \cap \hat{M}_{k-1}=\cap_{j=0}^{r} U_{k-1}\left(\omega_{j}\right)=U_{k-1}(\Phi)=U_{k}(\Phi) \cap \hat{M}_{k-1}$.

b) let $i \in I$ such that $\partial E_{i} \subseteq U_{k-1}\left(\omega_{j}\right)$ for every $j=0,1, \ldots, r$. Then $\partial E_{i} \subseteq$ $\cap_{j=0}^{r} U_{k-1}\left(\omega_{j}\right)=U_{k-1}(\Phi)$, and, consequently,

$$
\left(\cap_{j=0}^{r} U_{k}\left(\omega_{j}\right)\right) \cap E_{i}=E_{i}=U_{k}(\Phi) \cap E_{i} .
$$

c) Let $i \in I$ such that there exists a $j \in\{0,1, \ldots, r\}$ with $\partial E_{i} \nsubseteq U_{k-1}\left(\omega_{j}\right)$. then $\partial E_{i} \nsubseteq U_{k-1}(\Phi)$, and, consequently,

$$
\begin{aligned}
\left(\cap_{j=0}^{r} U_{k}\left(\omega_{j}\right)\right) \cap E_{i} & =g_{i}\left(\left\{\lambda x \mid 0<\lambda \leq 1 \text { and } x \in f_{i}^{-1}\left(\cap_{j=0}^{r} U_{k-1}\left(\omega_{j}\right)\right)\right\}\right) \\
& =g_{i}\left(\left\{\lambda x \mid 0<\lambda \leq 1 \text { and } x \in f_{i}^{-1}\left(U_{k-1}(\Phi)\right)\right\}\right) \\
& =U_{k}(\Phi) \cap E_{i} .
\end{aligned}
$$

a), b) and c) show that $\bigcap_{j=0}^{r} U_{k}\left(\omega_{j}\right)=U_{k}(\Phi)$.

7) Let $e \in \hat{M}_{k}$. If $e \in \hat{M}_{k-1}$, then, by the inductive hypothesis, there exists a vertex $\omega$ of $\operatorname{Del}(\mathscr{A})$ such that $e \in U_{k-1}(\omega) \subseteq U_{k}(\omega)$. Assume now that there exists an $i \in I$ such that $e \in E_{i}$. Let $\Phi$ denote the simplex of $\operatorname{Del}(\mathscr{A})$ such that $i \in I_{\Phi}$. By the construction of $\hat{M}_{k}$, we have $\partial E_{\imath} \subseteq U_{k-1}(\Phi)$, and, by the construction of $U_{k}(\Phi)$, we have $e \in E_{i} \subseteq U_{k}(\Phi)$. By Property 6), $e \in U_{k}(\omega)$, where $\omega$ is any vertex of $\Phi$.

Now , we set:

a) $\hat{M}_{\infty}=\lim \hat{M}_{k}$

b) $U_{\infty}(\Phi) \stackrel{\rightarrow}{=} \lim U_{k}(\Phi)$ for every simplex of $\operatorname{Del}(\mathscr{A})$

We have the following properties.

1) $\pi_{n}\left(\hat{M}_{\infty}\right)=\pi_{n}(\hat{M}(\mathscr{A}))$ for every $n \in\left\{0,1, \ldots, n_{0}-1\right\}$, and $\pi_{n}\left(\hat{M}_{\infty}\right)=$ $\pi_{n}\left(\hat{M}_{n}\right)$ for every $n \geq n_{0}$.

2) $\pi_{n}\left(U_{\infty}(\Phi)\right)=\{0\}$ for every $n \geq 0$ and for every simplex $\Phi$ of $\operatorname{Del}(\mathscr{A})$.

3) Let $\omega_{0}, \omega_{1}, \ldots, \omega_{r}$ be $(r+1)$ vertices of $\operatorname{Del}(\mathscr{A})$. If $\cap_{j=0}^{r} U_{\infty}\left(\omega_{j}\right) \neq \varnothing$, then $\omega_{0}, \omega_{1}, \ldots, \omega_{r}$ are the vertices of a simplex $\Phi$ of $\operatorname{Del}(\mathscr{A})$.

4) Let $\omega_{0}, \omega_{1}, \ldots, \omega_{r}$ be the vertices of a simplex $\Phi$ of $\operatorname{Del}(\mathscr{A})$. Then $\cap_{j=0}^{r} U_{\infty}\left(\omega_{j}\right)=U_{\infty}(\Phi)$.

5) $U_{\infty}=\left\{U_{\infty}(\omega) \mid \omega\right.$ a vertex of $\left.\operatorname{Del}(\mathscr{A})\right\}$ is a covering of $\hat{M}_{\infty}$ by open subsets. 
Properties 3), 4) and 5) show that $\boldsymbol{U}_{\infty}$ is a covering of $\hat{M}_{\infty}$ having $\operatorname{Del}(\mathscr{A})$ as nerve. Properties 2) and 4) show that any nonempty intersection of elements of $\boldsymbol{U}_{\infty}$ is contractible. It follows, by [We], that $\operatorname{Del}(\mathscr{A})$ is homotopically equivalent to $\hat{M}_{\infty}$.

Since $\pi_{n_{0}}\left(\hat{M}_{\infty}\right)=\pi_{n_{0}}\left(\hat{M}_{n_{0}}\right)$ and the inclusion map $\hat{M}(\mathscr{A}) \rightarrow \hat{M}_{n_{0}}$ induces a surjective morphism $\xi_{n_{0}}: \pi_{n_{0}}(\hat{M}(\mathscr{A})) \rightarrow \pi_{n_{0}}\left(\hat{M}_{n_{0}}\right)$, in order to prove that $\operatorname{Del}(\mathscr{A})$ is not homotopically equivalent to $\hat{M}(\mathscr{A})$, it suffices to show that $\xi_{n_{0}}$ is not injective.

Choose a simplex $\Phi^{o}$ of $\operatorname{Del}^{o}(\mathscr{A})$ such that $\pi_{n_{0}}\left(U\left(\Phi^{o}\right)\right) \neq\{0\}$. Let $\hat{\iota}_{\Phi^{o}}^{0}$ : $U\left(\Phi^{\circ}\right) \rightarrow \hat{M}(\mathscr{A})$ be the inclusion map of $U\left(\Phi^{\circ}\right)$ into $\hat{M}(\mathscr{A})$, and let $\hat{\iota_{\Phi}}: \hat{M}(\mathscr{A}) \rightarrow$ $\hat{M}\left(\mathscr{A}_{X\left(\Phi^{o}\right)}\right)$ be the map defined in the proof of Lemma 3.8. Then $\hat{\iota}_{\Phi^{o}}=\hat{\iota}_{\Phi^{o}} \circ \hat{\imath}_{\Phi^{o}}^{0}$ is a homotopy equivalence (see the proof of Lemma 3.8 ), thus $\left(\hat{\ell}_{\Phi^{o}}^{0}\right)_{*}: \pi_{n_{0}}\left(U\left(\Phi^{o}\right)\right) \rightarrow$ $\pi_{n_{0}}(\hat{M}(\mathscr{A}))$ is injective. Furthermore, by construction of $\hat{M}_{n_{0}}$, the morphism $\xi_{n_{0}}$ 。 $\left(\hat{\iota}_{\Phi^{o}}^{0}\right)_{*}: \pi_{n_{0}}\left(U\left(\Phi^{o}\right)\right) \rightarrow \pi_{n_{0}}\left(\hat{M}_{n_{0}}\right)$ sends $\pi_{n_{0}}\left(U\left(\Phi^{o}\right)\right)$ onto $\{0\}$. This shows that $\xi_{n_{0}}$ is not injective.

\section{REFERENCES}

[De] P. Deligne, Les immeubles des groupes de tresses généralisés, Invent. Math., 17 (1972), 273-302

[FR] M. Falk \& R. Randell, On the homotopy theory of arrangements, in "Complex Analytic Singularities," Advanced Studies in Pure Math. 8, North Holland, 1987 pp. $101-124$

[LW] A.T. Lundell \& S. Weingram, "The topology of CW-complexes," Van Nostrand Reinhold Company, New York, 1969.

[Or] P. Orlik, "Introduction to arrangements," CBMS Lecture Notes 72, Amer. Math. Soc., 1989

[OT] P. Orlik \& H. Terao, "Arrangements of hyperplanes," Springer-Verlag, New York, 1992

[Pa1] L. Paris, The covers of a complexified real arrangement of hyperplanes and their fundamental groups, Topology and its Applications, to appear.

[Pa2] - Universal cover of Salvetti's complex and topology of simplicial arrangements of hyperplanes, Trans. Amer. Math. Soc., to appear.

[We] A. Weil, Sur les theoremes de De Rham, Comm. Math. Helv., 26 (1952), 119-145.

Département de Mathématiques

Universite de Nantes

2 rue de la Houssinière

44072 Nantes Cedex 03

France 